\title{
Data Driven Based Method for Field Information Sensing
}

\author{
Jing Lei ${ }^{1,2}$ \\ ${ }^{1}$ School of Energy, Power and Mechanical Engineering, North China Electric Power University, Changping District, \\ Beijing 102206, China \\ ${ }^{2}$ Key Laboratory of Efficient Utilization of Low and Medium Grade Energy (Tianjin University), \\ Ministry of Education of China, Tianjin 300072, China \\ Correspondence should be addressed to Jing Lei; leijing2002@gmail.com
}

Received 15 July 2014; Accepted 16 October 2014; Published 9 November 2014

Academic Editor: Massimo Scalia

Copyright (C) 2014 Jing Lei. This is an open access article distributed under the Creative Commons Attribution License, which permits unrestricted use, distribution, and reproduction in any medium, provided the original work is properly cited.

Acquiring the field information on temperature, pressure, concentration, or velocity is crucial for the monitoring of chemical reactors, multiphase flow systems, heat transfer units, atmospheric pollutants diffusion, and underground pollutant migration. In this paper, a dimensionality reduction matrix completion (DRMC) method is proposed for the field information sensing (FIS) of objects of interest from the scattered point measurement data. An objective functional that casts the FIS task as an optimization problem is proposed. An iteration scheme is developed for solving the proposed objective functional. Numerical simulations are implemented to validate the feasibility and effectiveness of the proposed algorithm. It is found that differing from common inverse problems, numerical simulation approaches, and tomography based field measurement methods, in the proposed method the field information can be reconstructed without the knowledge on governing equations of the measurement objects, initial conditions, boundary conditions, and physical properties of materials, except the limited number of the measurement data. As a result, an alternative insight is introduced for the FIS problems.

\section{Introduction}

Acquiring the field information on temperature, pressure, concentration, or velocity is crucial for the monitoring of chemical reactors, multiphase flow systems, heat transfer units, and the underground pollutant migration and thus facilitates the improvement of the system efficiency, the energy saving, and the reduction of the pollutant emission. With the increasing concerns of the energy and environment problems, especially, the key issue on the monitoring of the large-scale objects has attracted wide attention. In order to satisfy the above requirements, two kinds of approaches, including numerical simulation methods and measurement approaches, are available. Owing to the challenges, such as (1) the understanding of the complicated mechanisms of the underlying process and the acquisition of the reasonable mathematical model are challenging, (2) it is hard to provide reliable governing equations, boundary conditions, physical property parameters, and initial conditions due to the restrictions of real conditions and (3) numerical simulations are time-consuming; applying numerical simulation methods to achieve the monitoring and control of the large-scale dynamic objects may be impractical. Currently, different measurement methods, which can be approximately divided into two categories, such as point measurement methods and field measurement techniques, have been developed for acquiring such information. The point measurement methods can acquire the local measurement information. However, it is hard for point measurement methods to describe the comprehensive situation of the measurement objects. The field measurement methods can acquire the field information of the state variables of interest. However, the costs of the measurement methods are often higher owing to the additional devices. In particular, common tomographybased field measurement methods require the closure of the sensor array, which may be inappropriate for the large-scale measurement objects such as the atmospheric pollutants diffusion, the wind field reconstruction, the large-scale temperature distribution measurement, and the monitoring of the underground pollutant migration. In common tomographybased measurement method, additionally, the sensitivity of the sensing in the measurement domain may gradually 
decrease with the increase of the scale of the measurement domain, which restricts the improvement of the measurement accuracy. With the development of the wireless sensor networks technologies and the point measurement methods, it is possible to acquire a large number of the scattered point measurement information. In order to address the above challenges, naturally, one of main motivations in this paper is to seek a reliable method to reconstruct the field information from finite observation data.

Mathematically speaking, the FIS problem can be described as follows: for a given field, which can be formulated as a matrix $\mathbf{X} \in \mathbb{R}^{n_{1} \times n_{2}}$, in which some entries are known and the remains are missing. The FIS problem tries to recover the matrix $\mathbf{X}$ with missing data from a sampling set $\mathbf{X}_{i, j}$, $(i, j) \in \Omega$, where $\Omega$ is a subset of the complete set with size $n_{1} \times n_{2}$. It is worth emphasizing that differing from common measurement approaches and numerical methods, the FIS method is a data driven measurement data, which integrates scattered measurement method and numerical optimization approach. Presently, different methods, including the artificial neural network (ANN) technique [1], the gappy proper orthogonal decomposition (GPOD) method or the principle component analysis (PCA) technique [2-5], the compressed sensing (CS) method [6], and the matrix completion (MC) method $[7,8]$, are available for the FIS problem. The ANN method belongs to the data driven approaches and has found wide applications in various fields. In real applications, in order to obtain a satisfactory result, the ANN method often requires a large number of the training samplings, which may be impractical for the FIS problem since the number of the observation data is commonly small. Furthermore, determining a suitable network structure is not a trivial task in real applications, which restricts applications of the ANN method. Additionally, the ANN method fails to exploit the prior information of the reconstruction objects, and thus the improvement of the reconstruction quality is restricted. One of the distinct characteristics in the PCA technique is that the data representation is not purely additive and each principle component consists of both negative and positive entries. It is worth mentioning that applying the GPOD algorithm or the PCA method to the FIS tasks requires a large number of snapshots to compute the basis vectors, which may be impractical owing to the uncertainties of the measurement objects and the lack of the understanding of the physical or chemical mechanisms of the underlying process. In the case of the large-scale monitoring especially, the above-mentioned challenges are more serious. The CS method is based on the fact that a relatively small number of the projections of a sparse signal can contain most of its salient information, which can be reconstructed by an appropriate algorithm. The CS method belongs to the direct reconstruction method, and the reconstruction results are far from satisfactory when the number of the measurement data is small. More importantly, in the CS method the missing data is directly reconstructed, and the computational load is heavy. The MC method is a procedure for recovering an unknown matrix with low rank or approximately low rank constraints from a small sampling of its entries. In other words, given a matrix with some unknown entries, the MC method tries to recover these unknown entries, such that the reconstructed matrix satisfies low rank or approximate low rank properties. This problem is motivated by the increasing requirements of inferring global structure from a small number of local observations, which has become an important research topic in many areas of engineering and science. It is found that the essence of the MC method is consistent with the FIS problem. In recent years, the MC method has been proposed for the FIS problems in other related fields. Like common direct reconstruction methods, unfortunately, the MC method performs poorly when the missing rate grows high. Like the CS method, especially, the MC method belongs to the direct reconstruction method, and the computational load is high when the large-scale monitoring problems are considered. From the viewpoint of real applications, a feasible FIS method should include at least two respects: acquiring satisfactory reconstruction results under a relatively small sampling numbers and a good robustness to the measurement noises.

Differing from common point measurement methods and tomography-based field measurement approaches, in order to resolve the above challenges, this paper presents a RDMC method that integrates the finite measurement information and mathematical methods for the FIS problem, which does not require information on governing equations, initial conditions, boundary conditions, physical properties of materials, and additional and costly measurement devices, but finite point measurement data. In the proposed method, especially, the number of the unknown variables depends only on that of the measurement data, which will facilitate real applications since the number of the measurement data is always small. The main contributions of the paper can be summarized as follows.

(1) Differing from common measurement techniques or numerical simulation methods, a DRMC method is proposed for the FIS problem, which does not require the information on governing equations of the measurement objects, initial conditions, boundary conditions, and physical property parameters of materials, except the limited number of the measurement data.

(2) An objective functional that casts the FIS task as an optimization problem is proposed. An iteration scheme is developed for solving the proposed objective functional.

(3) This paper presents a general framework for the FIS problem from the partial observation data, which may be useful for other related problems.

Compared with common measurement techniques and numerical simulation methods, the significant properties of the proposed DRMC based FIS method can be outlined as follows.

(1) As compared to common inverse problems [9-16], with an aim of estimating the missing information from partial measurement data under the known conditions, including governing equations, boundary conditions, physical property parameters, or initial conditions, in the DRMC method the measurement information is used to estimate the comprehensive field information without any other additional conditions. Furthermore, the DRMC method does not solve the complicated governing equations. 
(2) As compared to common numerical simulation methods, such as the finite element method (FEM) and the finite difference method (FDM), the DRMC method acquires the field information without the knowledge on governing equations, initial conditions, boundary conditions, and physical property parameters of materials, except the limited number of the measurement data. It should be pointed out that acquiring the above-mentioned conditions that the implementations of the numerical computations require is not a trivial task owing to the restrictions of the real conditions and the lack of the adequate understandings of the underlying physical or chemical mechanisms of the dynamic behaviors of a dynamic object of interest.

(3) As compared to common tomography-based field measurement methods with the requirements of the additional and costly devices, the DRMC method only requires finite observation data that can be easily acquired by means of common point measurement methods. Common tomography-based measurement methods often require the closure of the sensor array, which may be inappropriate for the large-scale measurement objects such as the atmospheric pollutants diffusion, the wind field reconstruction, the large-scale temperature distribution measurement, and the monitoring of the underground pollutant migration. In common tomography-based measurement method, additionally, the sensitivity of the sensing in the measurement domain may decrease with the increase of the scale of the measurement domain, which restricts the improvement of the measurement accuracy. However, the DRMC method does not require such conditions, which is highly desirable for real applications.

(4) As compared to common point measurement techniques with a motivation of acquiring local measurement information, the DRMC method acquires the comprehensive field information from finite point measurement information.

(5) As compared to common CS method and the MC technique where the unknown variables are directly reconstructed, in the DRMC method the number of the unknown variables depends only on that of the measurement data.

The rest of this paper is organized as follows. In Section 2, the MC method is introduced. In Section 3, the DRMC model is proposed, an objective functional is designed to cast the FIS task as an optimization problem, and an iteration scheme is proposed to solve the proposed objective functional. A DRMC based FIS method is presented in Section 4. Numerical simulations are implemented to evaluate the feasibility of the proposed algorithm and the detailed discussions on the numerical results are provided in Section 5. Finally, Section 6 summarizes the main conclusions.

\section{Matrix Completion Method}

The FIS problem aims at reconstructing the filed information from finite measurement data. Mathematically speaking, the FIS problem can be described as follows: for a given twodimensional field that can be formulated as a matrix $\mathbf{X} \in$ $\mathbb{R}^{n_{1} \times n_{2}}$, in which some entries are known and the remains are missing. The FIS problem tries to recovery the matrix $\mathbf{X}$ with missing data from a sampling set $\mathbf{X}_{i, j},(i, j) \in \Omega$, where $\Omega$ is a subset of the complete set with size $n_{1} \times n_{2}$. In most cases, the number of the measurement data is far fewer than that of the missing data. The FIS problem is essentially an inverse problem, and it will be hard to solve without any additional information or constraints. It is found that matrices or fields that need to be recovered usually illustrate the low rank or approximately low rank properties in many instances. In the FIS task, the distributions of temperature, pressure, or concentration at different locations over different times present the correlations, which will obey specific physical or chemical mechanisms. Therefore, these data matrices will present low rank or approximately low rank properties owing to the inherent redundancy. According to the PCA method, in a low rank matrix the energy is always contributed by the top several singular values in real environments [17]. More discussions on the low rank property can be found in Candès and Recht [7], Candès and Tao [8], Candès and Plan [18], and Kong et al. [17]. In essence, the MC method is a procedure for recovering an unknown matrix with low rank or approximately low rank constraints from a sampling of its entries. Obviously, the motif of the MC method is consistent with the FIS problem.

Studies indicate that under some suitable conditions, one can recover an unknown low rank matrix from a nearly minimal set of entries by solving a simple convex optimization problem $[7,8,18]$. The main solution strategy for the MC method is based on the nuclear norm minimization. In a mathematical notation, the MC method can be generally formulated as $[7,8,18-23]$

$$
\begin{array}{cl}
\min & \{\operatorname{rank}(\mathbf{X})\} \\
\text { s.t. } & \mathbf{X}_{i, j}=\mathbf{M}_{i, j}, \quad(i, j) \in \Omega .
\end{array}
$$

We define a projection operator $P_{\Omega}(\cdot): \mathbb{R}^{n_{1} \times n_{2}} \rightarrow \mathbb{R}^{n_{1} \times n_{2}}$ as follows:

$$
\left(P_{\Omega}(\mathbf{A})\right)= \begin{cases}\mathbf{A}_{i, j}, & \text { if }(i, j) \in \Omega \\ 0, & \text { otherwise. }\end{cases}
$$

Submitting (2) to (1) yields

$$
\begin{array}{cl}
\min & \{\operatorname{rank}(\mathbf{X})\} \\
\text { s.t. } & P_{\Omega}(\mathbf{X})=P_{\Omega}(\mathbf{M}) .
\end{array}
$$

Directly solving (3) is not a trivial task. For the sake of easy computation, (3) can be relaxed as

$$
\begin{array}{cl}
\min & \left\{\|\mathbf{X}\|_{*}\right\} \\
\text { s.t. } & P_{\Omega}(\mathbf{X})=P_{\Omega}(\mathbf{M}),
\end{array}
$$

where operator $\|\cdot\|_{*}$ represents the nuclear norm for a matrix, and it can be specified as

$$
\|\mathbf{X}\|_{*}=\sum_{i=1}^{m} \sigma_{i}(\mathbf{X}),
$$

where $\sigma_{i}(\mathbf{X})$ defines the $i$ th largest singular value of matrix $\mathbf{X}$. 
According to the optimization theory and the Tikhonov regularization method, (4) can be cast as the following optimization problem [20]:

$$
\min \left\{\frac{1}{2}\left\|P_{\Omega}(\mathbf{M}-\mathbf{X})\right\|_{F}^{2}+\lambda\|\mathbf{X}\|_{*}\right\}
$$

where $\|\cdot\|_{F}$ represents the Frobenius norm and $\lambda>0$ can be called as the regularization parameter.

Equation (6) especially can be rewritten as the following concise expression [20]:

$$
\min \left\{\frac{1}{2}\|\boldsymbol{\psi} \mathbf{x}-\mathbf{y}\|^{2}+\lambda\|\mathbf{X}\|_{*}\right\}
$$

where $\mathbf{x}$ represents the vectorized version of the matrix $\mathbf{X} ; \psi$ is a restriction operator, and it has the elements as ones at the sampling locations and $y$ represents the measurement data.

Currently, the MC method has been intensively studied and found wide applications in various fields $[24,25]$. The MC method refers to abundant theoretical background, and more details and real applications can be found in Candès and Recht [7], Candès and Tao [8], Candès and Plan [18], Chen et al. [19], Majumdar and Ward [20], Majumdar [21], Toh and Yun [22], and Wen et al. [23]. Additionally, it should be pointed out that since the number of the missing data is large in the FIS problem, directly reconstructing the missing data is obviously impractical. In fact, the CS method and the MC technique suffer from such dilemma. Naturally, seeking a reliable method, in which the computational cost is independent of the number of the missing data, will be highly desired.

\section{Dimensionality Reduction Matrix Completion Method}

3.1. Reconstruction Model. In the case of the FIS problem, the number of the known measurement data is always smaller than that of the missing data, and directly reconstructing the missing data may be inappropriate. Naturally, seeking an efficient approach to reduce the number of the unknown variables is crucial. According to the radial basis function (RBF) method, an unknown function can be approximated as [26]

$$
f(\mathbf{x})=\sum_{j=1}^{M} \alpha_{j} \varphi\left(\left\|\mathbf{x}-\mathbf{x}_{j}\right\|\right)=\sum_{j=1}^{M} \alpha_{j} \varphi\left(\mathbf{r}_{j}\right),
$$

where $\mathbf{r}_{j}=\left\|\mathbf{x}-\mathbf{x}_{j}\right\|$ and $\varphi\left(\mathbf{r}_{j}\right)=\exp \left(-\mathbf{r}_{j}^{2} / 2 \sigma^{2}\right)$. It is worth mentioning that $\varphi\left(\mathbf{r}_{j}\right)$ can be predetermined according to a specific FIS task. In order to ensure the numerical stability especially, applying a polynomial $p(\mathbf{x})$ to (8) yields [26]

$$
\mathbf{y}=f(\mathbf{x})=\sum_{j=1}^{M} \boldsymbol{\alpha}_{j} \varphi\left(\left\|\mathbf{x}-\mathbf{x}_{j}\right\|\right)+\mathbf{p}(\mathbf{x}),
$$

where $\mathbf{p}(\mathbf{x})$ can be specified as

$$
\mathbf{p}(\mathbf{x})=\sum_{j=1}^{N} \boldsymbol{\beta}_{j} \mathbf{b}_{j}(\mathbf{x}),
$$

where $\boldsymbol{\beta}_{j}$ are unknown coefficients. It is worth emphasizing that in real applications $\mathbf{p}(\mathbf{x})$ can be designed according to a specific reconstruction task. For a two-dimensional problem, $\mathbf{b}$ can be specified as $\mathbf{b}=\{\mathbf{x}, \mathbf{y}, 1\}$ or $\mathbf{b}=\left\{\mathbf{x}, \mathbf{y}, \mathbf{x}^{2}, \mathbf{x y}, \mathbf{y}^{2}, 1\right\}$. Finally, submitting (10) to (9) yields the following expression:

$$
\mathbf{y}=f(\mathbf{x})=\sum_{j=1}^{M} \boldsymbol{\alpha}_{j} \varphi\left(\left\|\mathbf{x}-\mathbf{x}_{j}\right\|\right)+\sum_{j=1}^{N} \boldsymbol{\beta}_{j} \mathbf{b}_{j}(\mathbf{x})
$$

with an additional constraint on $\mathbf{p}(\mathbf{x})$ :

$$
\sum_{j=1}^{N} \boldsymbol{\alpha}_{j} \mathbf{b}_{j}\left(\mathbf{x}_{i}\right)=0
$$

It can be found from (11) that in order to estimate the missing data, the unknown variables $\boldsymbol{\alpha}$ and $\boldsymbol{\beta}$ need to be solved. In real FIS task, a measurement data set $\left\{\mathbf{x}_{i}, \mathbf{y}_{i}\right\}_{i=1}^{M}$ can be obtained. In this paper, for a two-dimensional problem, variables $\mathbf{b}$ and $\boldsymbol{\beta}$ are specified as $\mathbf{b}=\left[\mathbf{x}_{i}, \mathbf{y}_{i}, 1\right]^{T}$ and $\boldsymbol{\beta}=$ $\left[\boldsymbol{\beta}_{x}, \boldsymbol{\beta}_{y}, \boldsymbol{\beta}_{0}\right]^{T}$. Naturally, applying the known measurement data set to estimate the coefficients of (11) is suitable. For a known measurement data set, (11) can be reformulated as

$$
\left[\begin{array}{cccccc}
\boldsymbol{\varphi}_{1,1} & \cdots & \boldsymbol{\varphi}_{1, M} & \mathbf{x}_{1} & \mathbf{y}_{1} & 1 \\
\vdots & \vdots & \vdots & \vdots & \vdots & \vdots \\
\boldsymbol{\varphi}_{M, 1} & \cdots & \boldsymbol{\varphi}_{M, M} & \mathbf{x}_{M} & \mathbf{y}_{M} & 1 \\
\mathbf{x}_{1} & \cdots & \mathbf{x}_{M} & 0 & 0 & 0 \\
\mathbf{y}_{1} & \cdots & \mathbf{y}_{M} & 0 & 0 & 0 \\
1 & \cdots & 1 & 0 & 0 & 0
\end{array}\right]\left[\begin{array}{c}
\boldsymbol{\alpha}_{1} \\
\vdots \\
\boldsymbol{\alpha}_{M} \\
\boldsymbol{\beta}_{x} \\
\boldsymbol{\beta}_{y} \\
\boldsymbol{\beta}_{0}
\end{array}\right]=\left[\begin{array}{c}
\mathbf{y}_{1} \\
\vdots \\
\mathbf{y}_{M} \\
0 \\
0 \\
0
\end{array}\right]
$$

In (13), provided that the variables $\boldsymbol{\alpha}$ and $\boldsymbol{\beta}$ are solved, the missing data can be estimated by solving (11) using the location information of the missing data. For easy notation, (13) can be rewritten as the following system of the linear equations:

$$
\mathrm{AB}=\mathbf{Y}
$$

where

$$
\begin{gathered}
\mathbf{A}=\left[\begin{array}{cccccc}
\boldsymbol{\varphi}_{1,1} & \cdots & \boldsymbol{\varphi}_{1, M} & \mathbf{x}_{1} & \mathbf{y}_{1} & 1 \\
\vdots & \vdots & \vdots & \vdots & \vdots & \vdots \\
\boldsymbol{\varphi}_{M, 1} & \cdots & \boldsymbol{\varphi}_{M, M} & \mathbf{x}_{M} & \mathbf{y}_{M} & 1 \\
\mathbf{x}_{1} & \cdots & \mathbf{x}_{M} & 0 & 0 & 0 \\
\mathbf{y}_{1} & \cdots & \mathbf{y}_{M} & 0 & 0 & 0 \\
1 & \cdots & 1 & 0 & 0 & 0
\end{array}\right], \\
\mathbf{B}=\left[\begin{array}{c}
\boldsymbol{\alpha}_{1} \\
\vdots \\
\boldsymbol{\alpha}_{M} \\
\boldsymbol{\beta}_{x} \\
\boldsymbol{\beta}_{y} \\
\boldsymbol{\beta}_{0}
\end{array}\right], \\
\mathbf{Y}
\end{gathered}
$$

Equation (14) is an inverse problem, and integrating other prior information to the equation will facilitate the improvement of the quality of a solution. It is found that in the 
FIS problem, measurement objects, such as the temperature and concentration distributions, are in a dynamic evolution process, the measurement results at different time instants present the temporal correlations, and exploiting such prior information may be crucial for improving the reconstruction quality. In this paper, the DRMC method is proposed to exploit such prior information, which can be formulated as

$$
\begin{gathered}
\mathbf{B}_{k+1}=f\left(\mathbf{B}_{k}, \mathbf{v}_{k}\right) \\
\mathbf{A}_{k} \mathbf{B}_{k}=\mathbf{Y}_{k}+\mathbf{r}_{k},
\end{gathered}
$$

where $f$ describes the dynamic evolution information of the unknown variables, and it can be formulated as different equations according to different measurement objects; $\mathbf{v}_{k}$ represents the uncertainties of the dynamic evolution information and $\mathbf{r}_{k}$ defines the measurement noises. In order to achieve fast reconstruction, (16) is approximated by the following linearization formula:

$$
\mathbf{B}_{k+1}=\mathbf{F}_{k} \mathbf{B}_{k}+\mathbf{v}_{k}
$$

where $\mathbf{F}_{k}$ represents the state-transition operator at time instant $k$. If set $\mathbf{F}_{k}=\mathbf{I}$, where $\mathbf{I}$ is an identity matrix, (18) is a purely random-walk evolution model [27].

In (17), only the inaccurate properties on the measurement data are considered. However, it should be pointed out that applying the RBF method to approximate the unknown field variables will undoubtedly introduce deviations and thus simultaneously considering the inaccurate properties on the measurement data and the reconstruction model is crucial for the improvement of the estimation accuracy. With such considerations in mind, a dynamic reconstruction model that emphasizes the inaccurate properties on the measurement data and the reconstruction model is formulated as

$$
\mathbf{A}_{k} \mathbf{B}_{k}+\mathbf{E}_{k}=\mathbf{Y}_{k}+\mathbf{r}_{k}
$$

where $\mathbf{E}_{k}$ represents the model approximation deviations at the $k$ th instant.

Following the above discussions, when the linearization dynamic evolution equation is used, the DRMC method can be formulated as

$$
\begin{aligned}
& \mathbf{B}_{k+1}=\mathbf{F}_{k} \mathbf{B}_{k}+\mathbf{v}_{k} \\
& \mathbf{A}_{k} \mathbf{B}_{k}+\mathbf{E}_{k}=\mathbf{Y}_{k}+\mathbf{r}_{k} .
\end{aligned}
$$

3.2. Objective Functional. Equation (20) is an inverse problem, and directly solving the equation is not a trivial task. A popular approach is to cast the solving of the equation as an optimization problem by introducing the framework of the Tikhonov regularization method. In the Tikhonov regularization method, the objective functionals consist of the data fidelity and the constrained functionals that impose the prior constraints on the reconstruction objects [2830]. The design of the objective functional is crucial, which will influence the quality of a solution. In this paper, an objective functional that imposes the prior constraints on the underlying reconstruction objects is proposed, which can be specified as

$$
\begin{aligned}
\min \{ & \frac{1}{2}\left\|\mathbf{A}_{k} \mathbf{B}_{k}+\mathbf{E}_{k}-\mathbf{Y}_{k}\right\|^{2}+\frac{\mu_{1}}{2}\left\|\mathbf{B}_{k}-\mathbf{F}_{k} \mathbf{B}_{k-1}\right\|^{2} \\
+ & \frac{\mu_{2}}{2}\left\|\mathbf{B}_{k}-\mathbf{B}_{k-1}\right\|^{2} \\
& \left.+\frac{\mu_{3}}{2}\left\|\mathbf{E}_{k}\right\|^{2}+\mu_{4}\left\|\left(\mathbf{R B}_{k}\right)_{\text {mat }}\right\|_{*}+\frac{\mu_{5}}{2}\left\|\mathbf{W}_{k} \mathbf{B}_{k}\right\|^{2}\right\},
\end{aligned}
$$

where $\mu_{1}>0, \mu_{2}>0, \mu_{3}>0, \mu_{4}>0$, and $\mu_{5}>$ 0 are called the regularization parameters; $\left\|\mathbf{W}_{k} \mathbf{B}_{k}\right\|^{2}$ is the spatial constraint, in which $\mathbf{W}_{k}$ stands for a predetermined weighted matrix; $\left\|\mathbf{B}_{k}-\mathbf{F}_{k} \mathbf{B}_{k-1}\right\|^{2}$ is used to fuse the dynamic evolution information of the unknown variables; $\| \mathbf{B}_{k}-$ $\mathbf{B}_{k-1} \|^{2}$ defines the temporal constraint, which is introduced to emphasize the temporal correlations of a dynamic object; $\left(\mathbf{R B}_{k}\right)_{\text {mat }}$ represents a matrix that is formed by rearranging the vector $\mathbf{R B}_{k} ;\left\|\left(\mathbf{R B}_{k}\right)_{\text {mat }}\right\|_{*}$ is employed to impose the low rank constraint of the reconstruction objects, in which $\mathbf{R}$ can be formed according to (11).

The appealing properties of (21) can be outlined as follows.

(1) Equation (21) integrates the dynamic evolution information of the measurement objects and the observation information, which will facilitate the improvement of the reconstruction quality because of the increase of the quantity of information.

(2) In (21), the unknown field information is indirectly reconstructed by solving a low-dimensional coefficient vector, the dimensionality of the original unknown variables is reduced, and thus the computational cost is decreased.

(3) It is found that the field information is often in low rank owing to the spatial redundancies and temporal correlations of the underlying measurement objects. In (21), such prior information is exploited by introducing the nuclear norm. It is worth emphasizing that in (21) the dimensionality reduction and the low rank property of the reconstruction objects are simultaneously achieved, which is distinctly different from common MC method.

(4) The inaccurate properties on the measurement equation and the dynamic evolution equation of the measurement objects are emphasized in (21). Particularly, the inaccurate properties of the measurement data and the reconstruction model in the measurement equation are simultaneously considered, which is highly appropriate for real FIS tasks since such inaccuracies are ubiquitous.

(5) In practice, the measurement objects are often in a dynamic evolution process and the measurement results at different time instants present the temporal correlations. In (21), the temporal constraint is introduced to utilize the prior information, which is distinctly different from existing FIS methods.

3.3. Algorithm Structure. Equation (21) is an unconstraint optimization problem. For the sake of easy computation, following the computational strategies presented in Blondel et al. [31], Chan and Wong [32], Kim et al. [33], Lv et al. [34], 
Qin et al. [35], Tseng [36], and Xiao et al. [37], it is numerically appealing to decouple (21) as

$$
\begin{gathered}
\mathbf{E}_{k}^{\tau+1}=\min \left\{\frac{1}{2}\left\|\mathbf{A}_{k} \mathbf{B}_{k}^{\tau}+\mathbf{E}_{k}-\mathbf{Y}_{k}\right\|^{2}+\frac{\mu_{3}}{2}\left\|\mathbf{E}_{k}\right\|^{2}\right\} \\
\mathbf{B}_{k}^{\tau+1}=\min \left\{\frac{1}{2}\left\|\mathbf{A}_{k} \mathbf{B}_{k}+\mathbf{E}_{k}^{\tau+1}-\mathbf{Y}_{k}\right\|^{2}+\frac{\mu_{1}}{2}\left\|\mathbf{B}_{k}-\mathbf{F}_{k} \mathbf{B}_{k-1}\right\|^{2}\right. \\
+\frac{\mu_{2}}{2}\left\|\mathbf{B}_{k}-\mathbf{B}_{k-1}\right\|^{2} \\
\left.+\mu_{4}\left\|\left(\mathbf{R B}_{k}\right)_{\text {mat }}\right\|_{*}+\frac{\mu_{5}}{2}\left\|\mathbf{W}_{k} \mathbf{B}_{k}\right\|^{2}\right\},
\end{gathered}
$$

where $\tau$ represents the index of iterations.

It can be found that (22) can be easily solved, and seeking a reliable method to solve (23) will be crucial. According to the optimization theory, (23) can be rewritten as a constrained optimization problem by introducing an additively equality constraint:

$$
\begin{aligned}
\min \{ & \frac{1}{2}\left\|\mathbf{A}_{k} \mathbf{B}_{k}+\mathbf{E}_{k}^{\tau+1}-\mathbf{Y}_{k}\right\|^{2}+\frac{\mu_{1}}{2}\left\|\mathbf{B}_{k}-\mathbf{F}_{k} \mathbf{B}_{k-1}\right\|^{2} \\
& +\frac{\mu_{2}}{2}\left\|\mathbf{B}_{k}-\mathbf{B}_{k-1}\right\|^{2} \\
& \left.+\mu_{4}\left\|\mathbf{d}_{k}\right\|_{*}+\frac{\mu_{5}}{2}\left\|\mathbf{W}_{k} \mathbf{B}_{k}\right\|^{2}\right\}
\end{aligned}
$$

s.t. $\quad \mathbf{d}_{k}=\left(\mathbf{R} \mathbf{B}_{k}\right)_{\text {mat }}$.

In order to solve (24), applying the SBI method [38-44] to (24) yields

$$
\begin{gathered}
\min \left\{\frac{1}{2}\left\|\mathbf{A}_{k} \mathbf{B}_{k}+\mathbf{E}_{k}^{\tau+1}-\mathbf{Y}_{k}\right\|^{2}+\frac{\mu_{1}}{2}\left\|\mathbf{B}_{k}-\mathbf{F}_{k} \mathbf{B}_{k-1}\right\|^{2}\right. \\
+\frac{\mu_{2}}{2}\left\|\mathbf{B}_{k}-\mathbf{B}_{k-1}\right\|^{2}+\mu_{4}\left\|\mathbf{d}_{k}\right\|_{*} \\
\left.+\frac{\mu_{5}}{2}\left\|\mathbf{W}_{k} \mathbf{B}_{k}\right\|^{2}+\frac{\mu_{6}}{2}\left\|\mathbf{d}_{k}-\left(\mathbf{R} \mathbf{B}_{k}\right)_{\mathrm{mat}}-\mathbf{H}_{k}^{\tau}\right\|_{F}^{2}\right\} \\
\mathbf{H}_{k}^{\tau+1}=\mathbf{H}_{k}^{\tau}-\left(\mathbf{d}_{k}^{\tau+1}-\left(\mathbf{R} \mathbf{B}_{k}^{\tau+1}\right)_{\mathrm{mat}}\right) .
\end{gathered}
$$

Similarly, for the sake of easy computation, (25) can be decoupled as

$$
\begin{aligned}
\mathbf{B}_{k}^{\tau+1}=\min \{ & \frac{1}{2}\left\|\mathbf{A}_{k} \mathbf{B}_{k}+\mathbf{E}_{k}^{\tau+1}-\mathbf{Y}_{k}\right\|^{2} \\
& +\frac{\mu_{1}}{2}\left\|\mathbf{B}_{k}-\mathbf{F}_{k} \mathbf{B}_{k-1}\right\|^{2}+\frac{\mu_{2}}{2}\left\|\mathbf{B}_{k}-\mathbf{B}_{k-1}\right\|^{2} \\
& \left.+\frac{\mu_{5}}{2}\left\|\mathbf{W}_{k} \mathbf{B}_{k}\right\|^{2}+\frac{\mu_{6}}{2}\left\|\mathbf{d}_{k}^{\tau}-\left(\mathbf{R B}_{k}\right)_{\text {mat }}-\mathbf{H}^{\tau}\right\|_{F}^{2}\right\}
\end{aligned}
$$

$$
\mathbf{d}_{k}^{\tau+1}=\min \left\{\mu_{4}\left\|\mathbf{d}_{k}\right\|_{*}+\frac{\mu_{6}}{2}\left\|\mathbf{d}_{k}-\left(\mathbf{R} \mathbf{B}_{k}^{\tau+1}\right)_{\text {mat }}-\mathbf{H}^{\tau}\right\|_{F}^{2}\right\} .
$$

Following the above discussions, finally, an iteration scheme can be designed for solving the DRMC model, which can be summarized as follows.

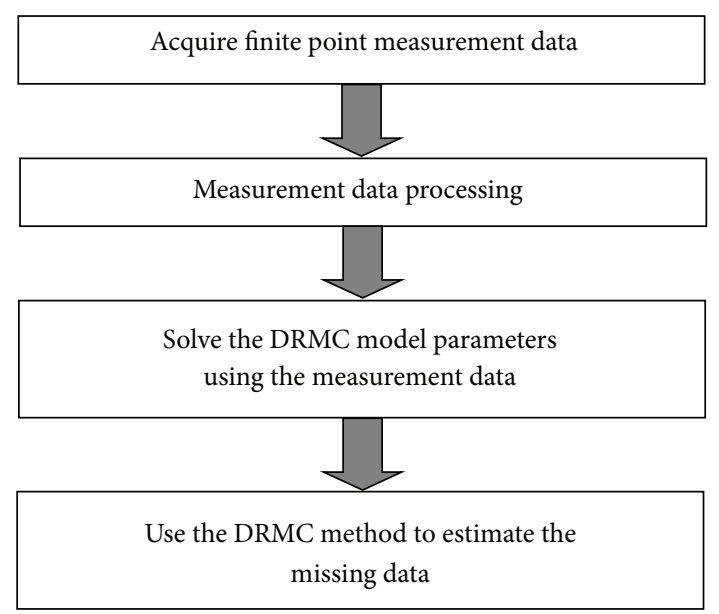

FIGURE 1: The DRMC method based FIS flowchart.

Step 1. Provide the input and output data, the algorithmic parameters, and the initial solutions.

Step 2. Set $k=1$.

Step 3. Determine the input data at the $k$ th instant.

Step 4. Update variable E by solving (22).

Step 5. Update variable B by solving (27).

Step 6. Update variable d by solving (28) using the singular value shrinkage operator [45].

Step 7. Update variable $\mathbf{H}$ according to (26).

Step 8. Loop to Step 4 until a predetermined iteration stopping criterion is satisfied.

Step 9. Set $k \leftarrow k+1$, loop to Step 3 until the maximum index of time is met.

\section{DRMC Method Based Field Information Sensing Procedure}

With the development of the point measurement methods and the wireless sensor networks technology, acquiring a large number of the scattered measurement information is possible. Therefore, applying the scattered measurement information to reconstruct the field information is appropriate. With such considerations in mind, in this section a DRMC based method is proposed for the FIS tasks, which can be outlined in Figure 1.

It can be found from Figure 1 that the DRMC method based FIS flowchart can be outlined as follows.

Step 1. The scattered measurement data is acquired by common measurement methods.

Step 2. Owing to the ubiquitous measurement noises, the raw measurement data is refined by the methods such as the wavelet based multiscale methods. 
Step 3. The measurement data is used to solve the DRMC model parameters; that is, solve (21) using the method presented in Section 3.3.

Step 4. Solve (11) to estimate the missing data.

It is worth emphasizing that compared with common measurement techniques and numerical simulation methods, the significant properties of the DRMC based FIS method can be summarized as follows.

(1) As compared to common inverse problems, in the DRMC method the measurement information is used to estimate the comprehensive field information without any other additional conditions, including governing equations, boundary conditions, physical property parameters, and initial conditions. It is worth emphasizing that the DRMC method does not solve complicated governing equations.

(2) As compared to common numerical simulation methods, such as the FEM and the FDM, the DRMC method acquires the field information without knowing governing equations, initial conditions, boundary conditions, and physical properties of materials, except the limited number of the observations.

(3) As compared to common tomography-based field measurement methods with the requirements of the additional and costly devices, the DRMC method does not require additional devices, but finite measurement data that can be easily obtained via common measurement methods.

(4) As compared to common point measurement techniques with a motivation of acquiring local measurement information, the DRMC method acquires the comprehensive field information from partial measurement information, which is highly attractive for real applications.

Similarly, compared with the MC method, the DRMC method has the following appealing properties.

(1) The DRMC method and the MC technique belong to the data driven methods, in which the estimation of the missing data does not require the knowledge on governing equations of underlying measurement objects, initial conditions, boundary conditions, and physical properties of materials.

(2) It is worth emphasizing that as compared to common MC method in which the unknown variables are directly reconstructed, the DRMC method reconstructs a low-dimensional coefficient vector rather than original unknown variables by introducing the dimensionality reduction method. It is found that the number of the unknown variables in the MC method is equivalent to that of the missing data. When the number of the unknown variables is large, the computational cost of the MC method is high. On the contrary, in the DRMC method the number of the unknown variables depends merely on that of the measurement data. Since the number of the measurement data is small in the FIS problems, the computational burden of the DRMC algorithm is smaller than the MC method.

(3) The DRMC method integrates the dynamic evolution information of the measurement objects and the measurement information. In the DRMC method, the temporal constraint is introduced to utilize the temporal correlations of the measurement objects, which is different from common MC method.

\section{Numerical Simulations and Discussions}

In previous sections, the DRMC model is proposed and an iteration scheme is developed for solving the model, and then the DRMC method based FIS flowchart is outlined. In this section, numerical simulations are implemented to evaluate the feasibility of the DRMC method. The simulation data is served as the measurement data. In order to simulate a real measurement environment, the simulation data is contaminated by the noises, which is defined as

$$
\mathbf{Y}_{\text {contaminated }}=\mathbf{Y}_{\text {original }}+\mathbf{r} \text {, }
$$

where $\mathbf{r}=\sigma_{1} \cdot \operatorname{rand} n ; \sigma_{1}$ represents the standard deviation and randn stands for a normal distribution random number with the mean of 0 and the standard deviation of 1 , which can be achieved by the function "randn" in the MATLAB software; $\mathbf{Y}_{\text {original }}$ and $\mathbf{Y}_{\text {contaminated }}$ define the original and noise-contaminated measurement data, respectively.

In all cases, the stopping criterion of iterations for the DRMC method and the MC algorithm is defined as $\left\|\mathbf{x}^{\tau+1}-\mathbf{x}^{\tau}\right\|^{2} /\left\|\mathbf{x}^{\tau+1}\right\|^{2} \leq 10^{-6}$, and the algorithmic parameters are approximately predetermined by solving (14) using the known observation data. In order to fairly evaluate the feasibility of the DRMC method, the purely random-walk evolution model is used to describe the dynamic evolution equation. All algorithms are implemented using the MATLAB 7.0 software on a PC with a Pentium IV $2.4 \mathrm{G} \mathrm{Hz}$ CPU and $4 \mathrm{G}$ bytes memory.

To evaluate the feasibility of the DRMC algorithm, the results reconstructed by the DRMC method are compared with the MC method. The mean relative error (MRE) is used to evaluate the quality of a solution, which can be defined as

$$
\eta=\frac{1}{n} \sum_{j=1}^{n}\left|\frac{\mathbf{X}_{\text {True }, j}-\mathbf{X}_{\text {Estimated }, j}}{\mathbf{X}_{\text {True }, j}}\right|
$$

where $\eta$ represents the MRE; operator $|\cdot|$ stands for the absolute value operator; $\mathbf{X}_{\text {True }}$ and $\mathbf{X}_{\text {Estimated }}$ represent the true values and the estimated values, respectively.

The sampling ratio is also used to evaluate the numerical performances of the DRMC method and the MC method, which is defined as

$$
\Phi=\frac{\text { The number of the samplings }}{\text { The number of the variables }} \times 100 \% \text {, }
$$

where $\omega$ represents the sampling ratio. 


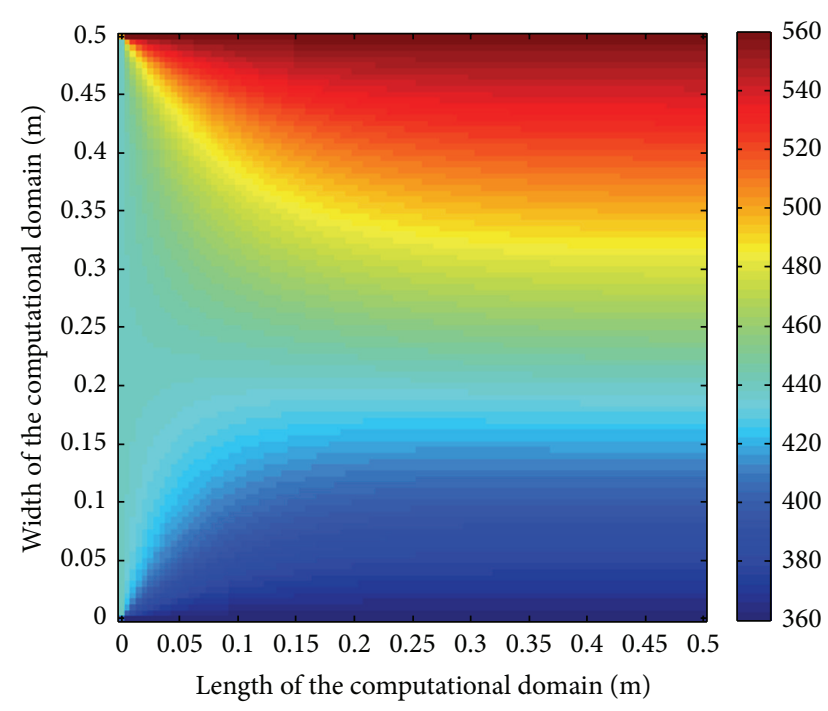

FIGURE 2: Original temperature distribution.

5.1. Case 1. In this section, the DRMC method is used to reconstruct the temperature distribution described by the following equations from the partial temperature measurement data:

$$
\begin{gathered}
\frac{\partial^{2} T}{\partial x^{2}}+\frac{\partial^{2} T}{\partial y^{2}}=0 \\
T(x, y)=440, \quad \text { when } x=0,0 \leq y \leq 0.5 \\
T(x, y)=560, \quad \text { when } y=0.5, \quad 0 \leq x \leq 0.5 \\
\frac{\partial T}{\partial x}=0, \quad \text { when } x=0.5,0 \leq y \leq 0.5 \\
T(x, y)=360, \quad \text { when } y=0,0 \leq x \leq 0.5 .
\end{gathered}
$$

The original temperature distribution is solved by the FEM, which is shown in Figure 2. In the MC method, $\alpha_{1}=50$. For the sake of easy computation, in the DRMC method, $\mathbf{W}_{k}=\operatorname{diag}\left(1 /\left|\mathbf{B}_{k-1, \tau, 1}\right|^{p}, 1 /\left|\mathbf{B}_{k-1, \tau, 2}\right|^{p}, \ldots, 1 /\left|\mathbf{B}_{k-1, \tau, n}\right|^{p}\right)$, $p=1.5, \mu_{1}=10^{-5}, \mu_{2}=10^{-5}, \mu_{3}=5, \mu_{4}=1, \mu_{5}$ $=1 \times 10^{-7}$, and $\sigma^{2}=0.01$. The simulation data is used to serve as measurement data, and the normal distribution random number with the variance of 9 is added to original data to simulate a real measurement environment. For fair comparison, in this case the known temperature data is randomly sampled. Figures 3 and 4 show the temperature distributions reconstructed by the DRMC algorithm and the MC method when the sampling ratio and the noise variance are $19.80 \%$ and 9 , respectively. Figure 5 presents the MREs of the MC method and the DRMC algorithm under different sampling ratios when the noise variance is 9 .

Figure 3 is the temperature distribution reconstructed by the DRMC method when the sampling ratio and the noise variance are $19.80 \%$ and 9 . It can be found from Figure 3 that because the proposed objective functional considers the inaccurate properties on the reconstruction

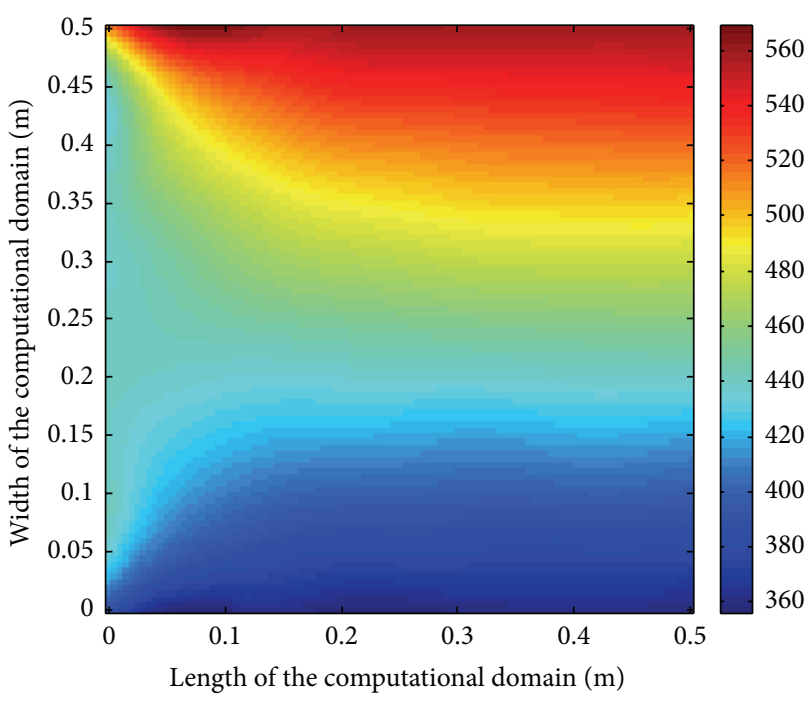

FIgURE 3: Temperature distribution reconstructed by the DRMC method when the noise variance and the sampling ratio are 9 and $19.80 \%$.

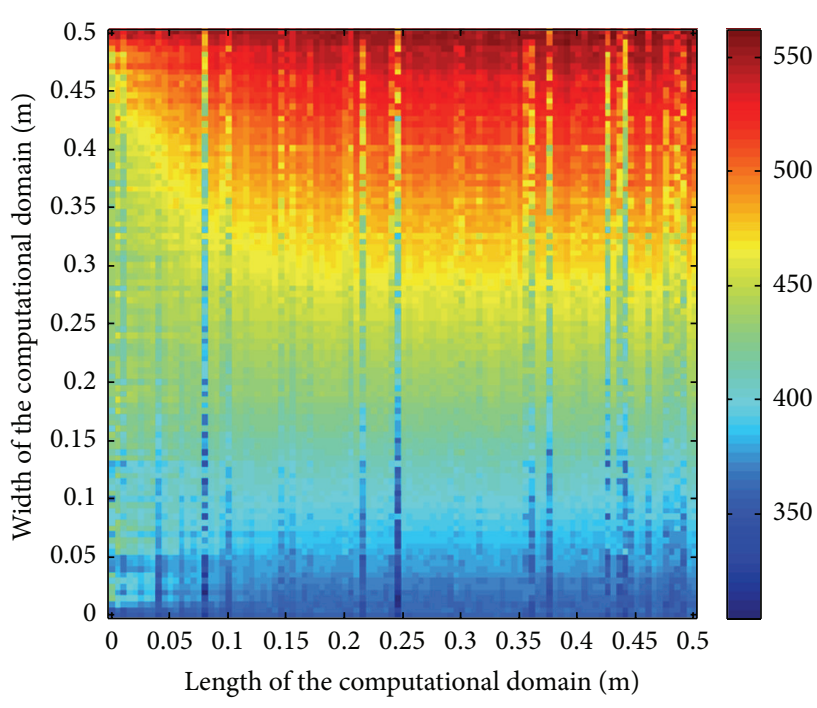

FIgURE 4: Temperature distribution reconstructed by the MC method when the noise variance and the sampling ratio are 9 and $19.80 \%$.

model and the measurement data, the dimensionality reduction of the unknown variables, and the low rank property of the reconstruction object, the temperature distribution reconstructed by the DRMC method is in a good agreement with the original distribution, and the MRE is $0.17 \%$ when the sampling ratio and the noise variance are $19.80 \%$ and 9 , which indicates that the DRMC method is successful in solving FIS problems.

The temperature distribution reconstructed by the MC method when the sampling ratio and the noise variance are $19.80 \%$ and 9 is shown in Figure 4. Numerical simulation results indicate that the implementation of the MC method is relatively easy and the low rank property of the reconstruction object is exploited. However, it can be observed from 


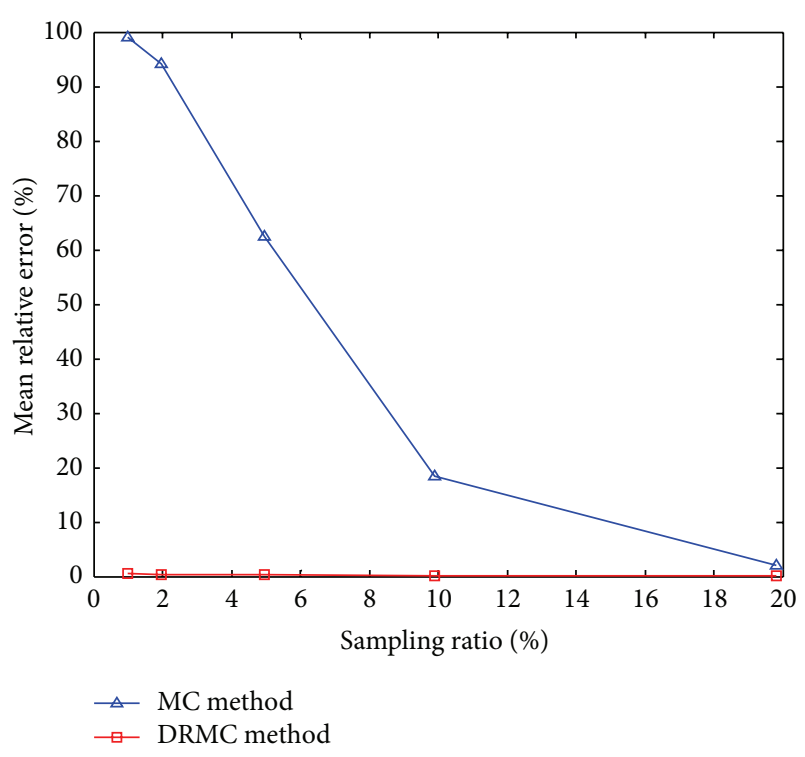

FIGURE 5: Mean relative errors under different sampling ratios when the noise variance is 9 .

Figure 4 that the reconstruction quality is not satisfactory. When the sampling ratio and the noise variance are $19.80 \%$ and 9 especially, the MRE of the MC method is $2.12 \%$, which is higher than the DRMC method.

When the noise variance is 9, the MREs for the DRMC method and the MC algorithm under different sampling ratios are shown in Figure 5. It is observed that with the increasing of the sampling ratios, the reconstruction quality of both algorithms is generally improved. However, it can be found from Figure 5 that the MC method performances poorly when the sampling ratios are relatively small. Additionally, it is worth mentioning that owing to the dimensionality reduction method is introduced to the DRMC method, the number of the unknown variables is equivalent to that of the measurement data, which is smaller than the number of the missing data, and thus a satisfactory estimation result can be obtained under the small sampling numbers. In fact, it can be found from Figure 5 that the reconstruction quality of the DRMC method is higher than that of the MC method when the sampling ratio is small. This feature is highly desired for real applications since the number of the measurement data is far smaller than that of the missing data.

It can be summarized from the above numerical results that as compared to common numerical simulation methods, such as the FEM and the FDM, the DRMC method acquires the field information without the knowledge on governing equations, initial conditions, boundary conditions, and physical properties of materials, except the limited number of the measurement data. As compared to common tomography-based field measurement methods with the requirements of the additional and costly devices, the DRMC method does not require additional devices but several measurement data that can be easily obtained via common measurement methods. As compared to common point measurement techniques, the DRMC method can

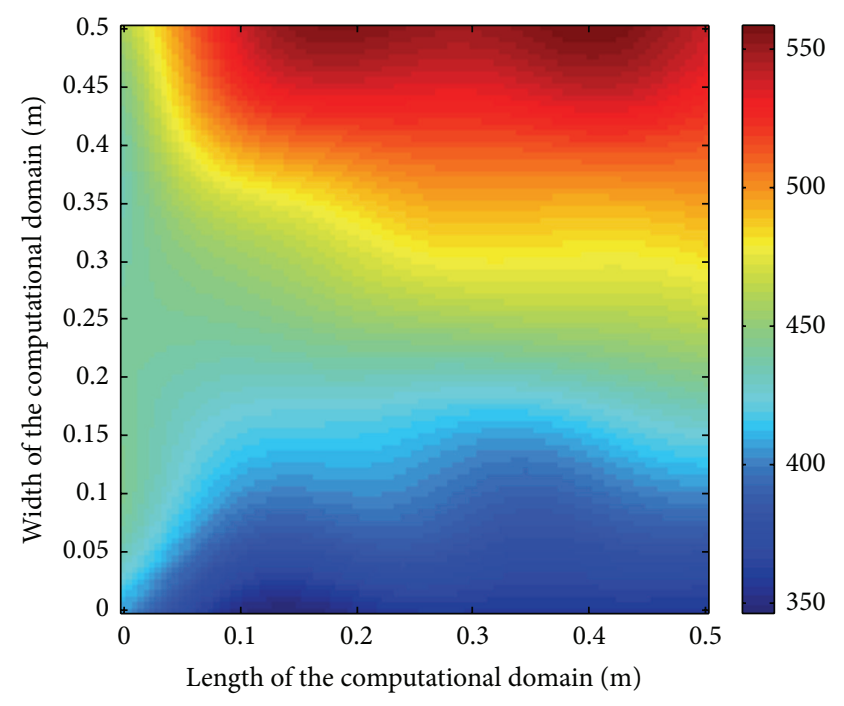

FIgURE 6: Temperature distribution reconstructed by the DRMC method when the noise variance and the sampling ratio are 25 and $0.90 \%$.

reconstruct the field information from partial observation data, which will facilitate the understanding of the underlying physical or chemical mechanisms of the dynamic behaviors of the measurement objects. Meanwhile, the low rank property of the measurement objects is imposed in the DRMC method, which will facilitate the improvement of the reconstruction quality. Additionally, it is worth mentioning that in the MC method and the CS based methods, the number of the unknown variables is equivalent to that of the missing data. When the number of the unknown variables is large, the lack of the known information is serious, and thus the improvement of the reconstruction quality is restricted. In the DRMC method the number of the unknown variables depends on that of the measurement data. Since the number of the measurement data is small in the FIS problems, the computational cost of the DRMC algorithm is smaller than the MC method and the CS based methods.

5.2. Case 2. In real applications, measurement noises are ubiquitous and complicated, a successful algorithm should be able to treat with the inaccurate properties of the measurement data. In this section, the noise-contaminated data with different variances is used to evaluate the robustness of the DRMC method. Figures 6, 7, and 8 show the temperature distributions reconstructed by the DRMC method when the noise variances are 25,100 , and 225 and the sampling ratio is $0.90 \%$, respectively. Figure 9 illustrates the MREs of the DRMC method under different noise variances.

The temperature distributions reconstructed by the DRMC method, when the noise variances are 25,100 , and 225 and the sampling ratio is $0.90 \%$, are shown in Figures 6-8, respectively. It can be found from Figures 6-9 that because the inaccurate properties on the reconstruction model and the measurement data are simultaneously considered, the DRMC method shows good robustness to the measurement noises, 


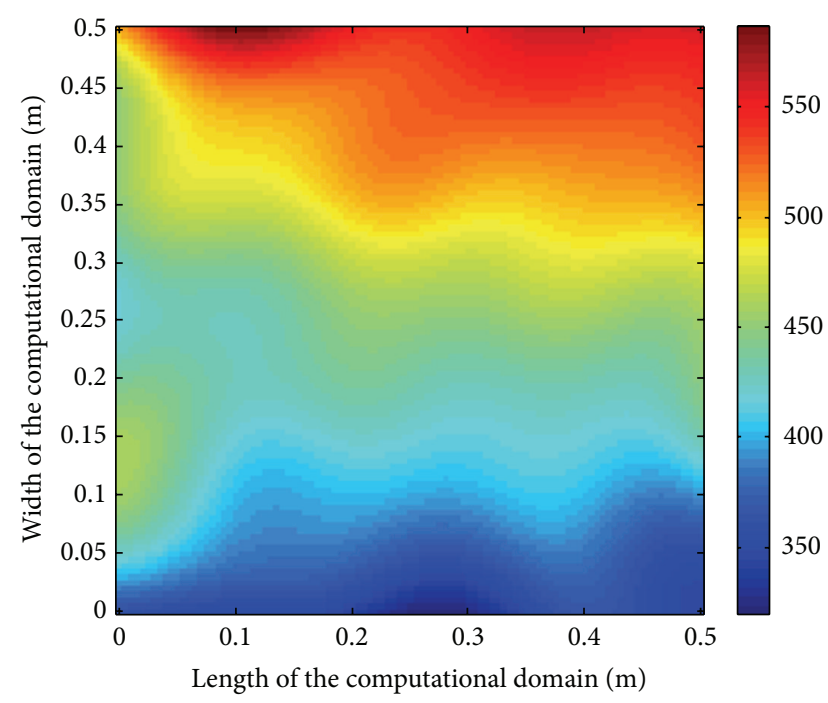

FIGURE 7: Temperature distribution reconstructed by the DRMC method when the noise variance and the sampling ratio are 100 and $0.90 \%$.

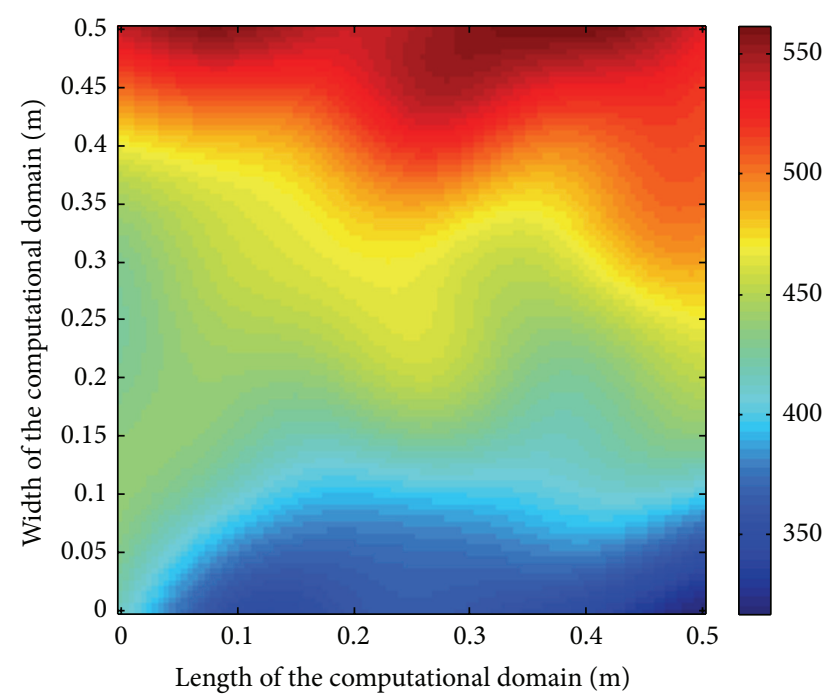

FIgURE 8: Temperature distribution reconstructed by the DRMC method when the noise variance and the sampling ratio are 225 and $0.90 \%$.

and the reconstruction results under different noise levels are in a good agreement with original temperature distributions. This advantage is highly desired for real applications since the measurement noises are ubiquitous and complicated.

5.3. Case 3. In order to further evaluate the feasibility of the DRMC method, another FIS problem is simulated. The original temperature distribution is described by

$$
\begin{gathered}
\frac{\partial^{2} T}{\partial x^{2}}+\frac{\partial^{2} T}{\partial y^{2}}=0 \\
T(x, y)=620, \quad \text { when } x=0,0 \leq y \leq 1
\end{gathered}
$$

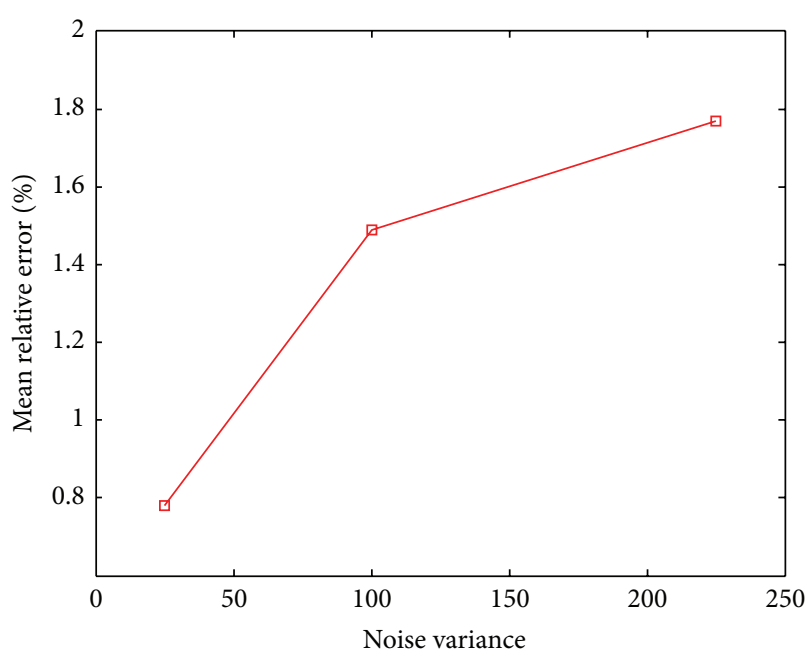

FIGURE 9: Mean relative errors under different noise variances.

$$
\begin{gathered}
-\lambda \frac{\partial T(x, y)}{\partial y}=h \times(T-273.15), \\
\text { when } y=1, \quad 0 \leq x \leq 1 \\
\frac{\partial T(x, y)}{\partial x}=0, \quad \text { when } x=1,0 \leq y \leq 1 \\
T(x, y)=420, \quad \text { when } y=0,0 \leq x \leq 1,
\end{gathered}
$$

where $\lambda=400$ and $h=100$.

In this case, the original temperature distribution, which is shown in Figure 10, is solved by the FEM. In the DRMC method, $\mathbf{W}$ is defined as an identity matrix, $\mu_{1}=10^{-5}, \mu_{2}=$ $10^{-5}, \mu_{3}=5, \mu_{4}=1, \mu_{5}=1 \times 10^{-7}$, and $\sigma^{2}=0.01$. In the MC method, $\alpha_{1}=50$. The simulation data is used to serve as the measurement data, and the random number of satisfying the normal distribution with the variance of 9 is added to original data to simulate a real measurement environment. Figures 11 and 12 show the temperature distributions reconstructed by the DRMC method and the MC method when the sampling ratio and the noise variances are $19.80 \%$ and 9 , respectively. When the noise variance is 9, the MREs for the DRMC method and the MC method under different sampling ratios are shown in Figure 13.

Figure 11 is the temperature distribution reconstructed by the DRMC method when the sampling ratio and the noise variance are $19.80 \%$ and 9 . As can be expected, it can be found from Figure 11 that the temperature distribution reconstructed by the DRMC method is in a good agreement with the original distribution and the MRE is $0.17 \%$, which indicates that the DRMC method is successful in solving the FIS task. When the sampling ratio and the noise variance are $19.80 \%$ and 9 , the temperature distribution reconstructed by the MC method is shown in Figure 12. It can be seen from Figure 12 that the reconstruction quality of the MC method is not satisfactory. When the sampling ratio and the noise 


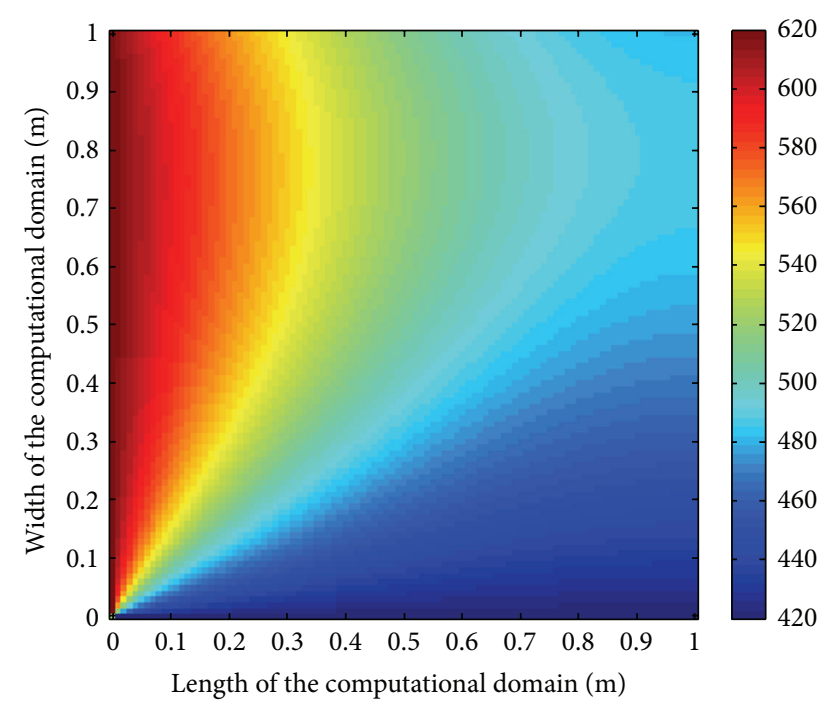

FIgURE 10: Original temperature distribution.

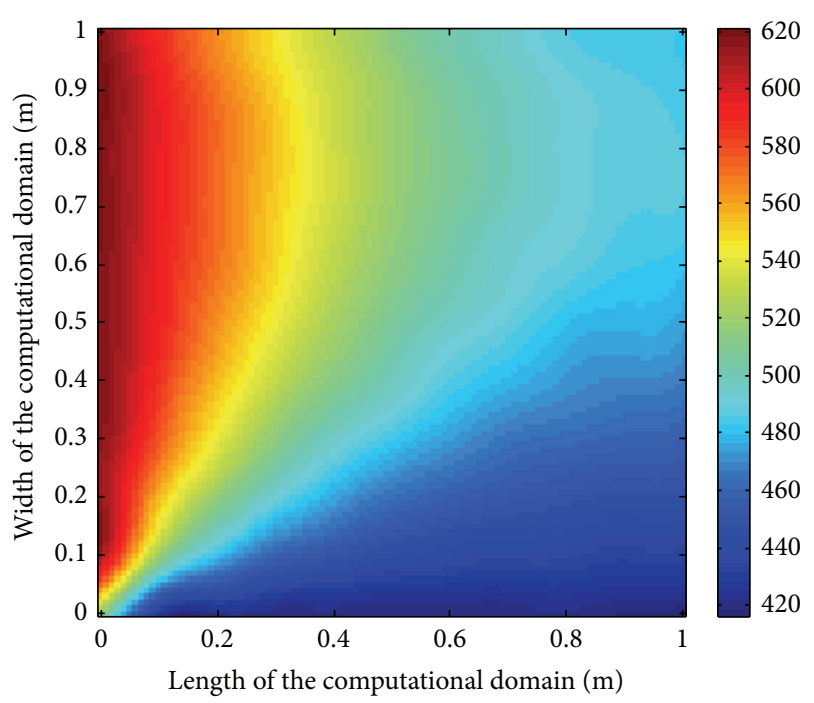

FIGURE 11: Temperature distribution reconstructed by the DRMC method when the noise variance and the sampling ratio are 9 and $19.80 \%$.

variance are $19.80 \%$ and 9, the MRE of the MC method is $2.95 \%$, which is higher than the DRMC method.

When the noise variance is 9, the MREs of the DRMC method and the MC method under different sampling ratios are shown in Figure 13. It can be observed that with the increasing of the sampling ratios the reconstruction quality of the both algorithms is generally improved. However, the reconstruction quality of the DRMC method is distinctly higher than that of the MC method when the sampling ratio is relatively small. This advantage is highly desired for real applications since the number of the measurement data is far smaller than that of the missing data.

5.4. Case 4. In order to further evaluate the numerical performances of the DRMC method, another FIS problem is

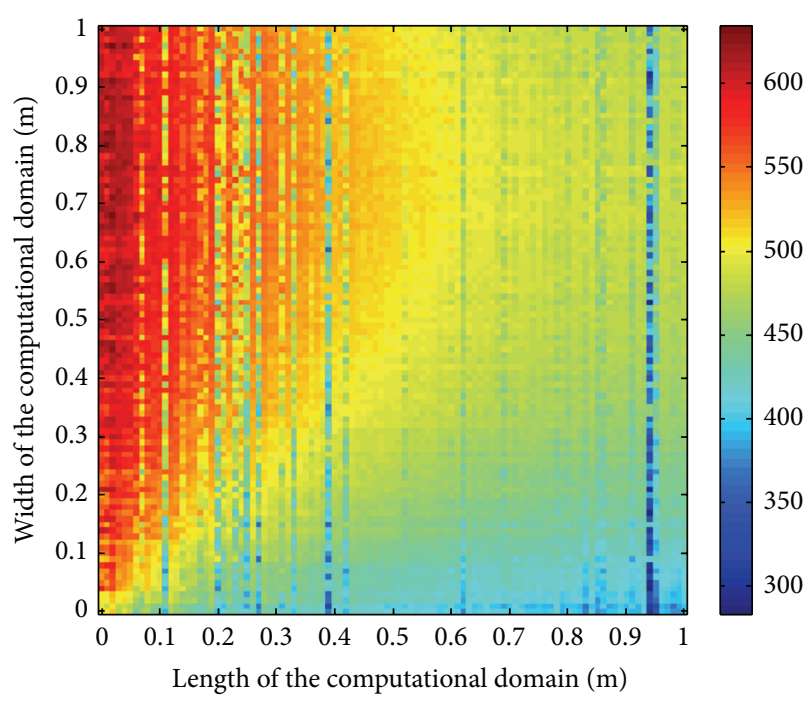

FIgURE 12: Temperature distribution reconstructed by the MC method when the noise variance and the sampling ratio are 9 and $19.80 \%$.

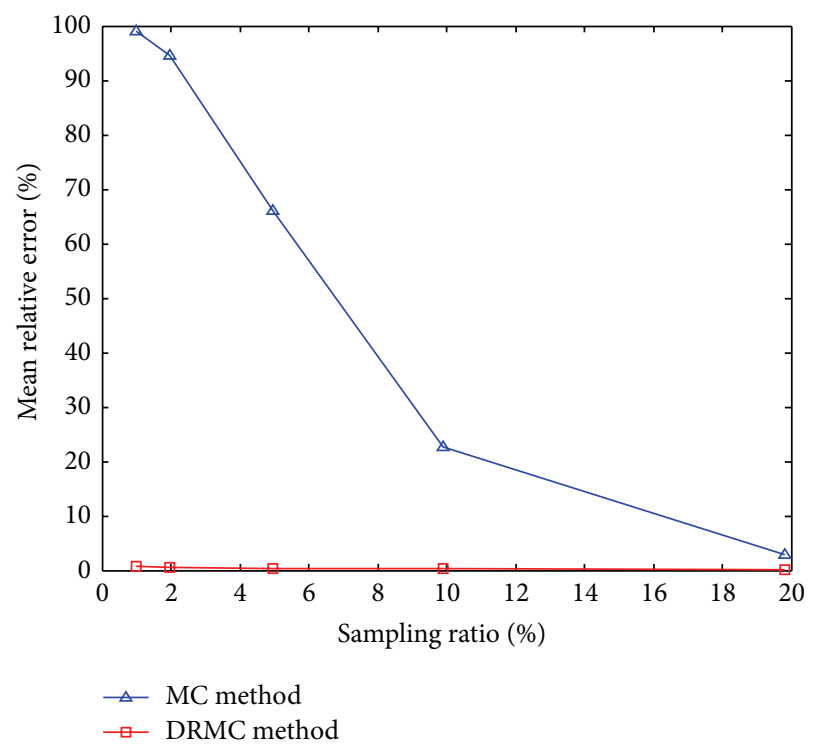

FIGURE 13: Mean relative errors under different sampling ratios.

simulated. In this section, the original temperature distribution is described by [46]

$$
\begin{aligned}
T(x, y)= & 1000 \times \exp \left(\frac{30(y-2)^{2}-10 x^{2}}{100}\right)+1000 \\
& \times \exp \left(\frac{-30(y+2)^{2}-10 x^{2}}{100}\right)+800 .
\end{aligned}
$$

Figure 14 presents the original temperature distribution derived from (34). In the $\mathrm{MC}$ method, $\alpha_{1}=$ 50. For the sake of easy computation, in the DRMC method, $\mathbf{W}_{k}$ is defined as a diagonal matrix $\mathbf{W}_{k}=$ $\operatorname{diag}\left(1 /\left|\mathbf{B}_{k-1, \tau, 1}\right|^{p}, 1 /\left|\mathbf{B}_{k-1, \tau, 2}\right|^{p}, \ldots, 1 /\left|\mathbf{B}_{k-1, \tau, n}\right|^{p}\right), p=1.5$, 


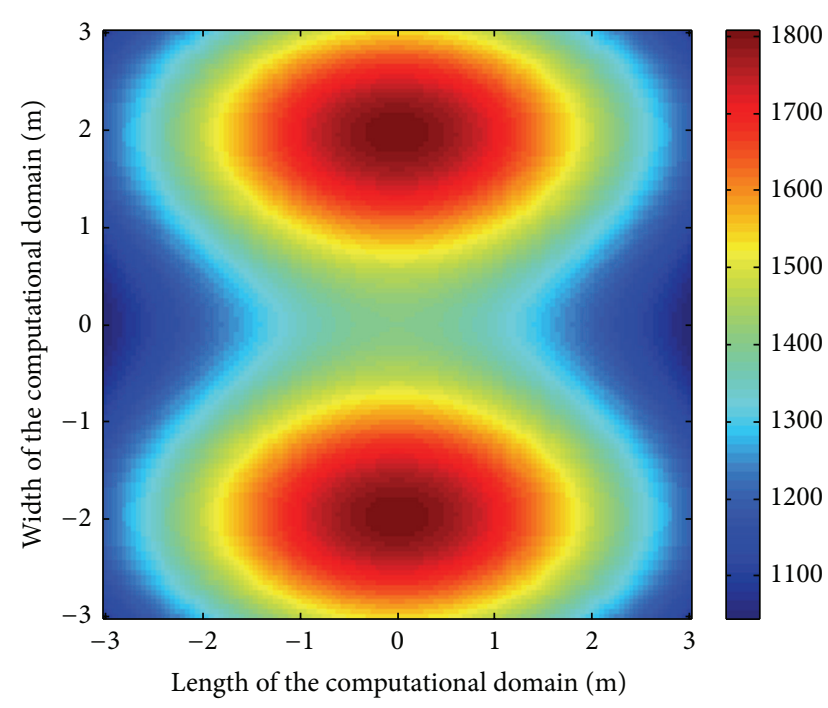

FIGURE 14: Original temperature distribution.

$\mu_{1}=10^{-5}, \mu_{2}=10^{-5}, \mu_{3}=5, \mu_{4}=1, \mu_{5}=1 \times$ $10^{-7}$, and $\sigma^{2}=1$. The simulation data is used to serve as the measurement data, and the normal distribution random number with the variance of 9 is added to original data to simulate a real measurement environment. Figures 15 and 16 show the temperature distributions reconstructed by the DRMC method and the MC method when the sampling ratio and the noise variances are $33.06 \%$ and 9 , respectively. When the variance of the noise is 9 , the MREs of the DRMC method and the MC method under different sampling ratios are illustrated in Figure 17.

Figures 15 and 16 are the temperature distributions reconstructed by the DRMC method and the MC algorithm when the sampling ratio and the noise variance are $33.06 \%$ and 9, respectively. As can be expected, it can be seen from Figure 15 that the temperature distribution is in a good agreement with the original distribution and the MRE is $0.025 \%$, which indicates that the DRMC method is successful in implementing the FIS task. Particularly, it can be observed from Figure 16 that the reconstruction quality of the MC method is not satisfactory, and the MRE is $10.41 \%$, which is higher than the DRMC method.

When the noise variance is 9, the MREs of the DRMC method and the MC method under different sampling ratios are shown in Figure 17. It can be seen that with the increasing of the sampling ratios the reconstruction quality of the both algorithms is improved. However, it can be found that the reconstruction quality of the DRMC method is higher than that of the MC method when the sampling ratio is small.

5.5. Case 5. In real applications, the measurement objects are in a dynamic evolution process. A reliable reconstruction algorithm should be able to deal with such measurement objects. In this section, the DRMC method is used to reconstruct the dynamic temperature distributions described

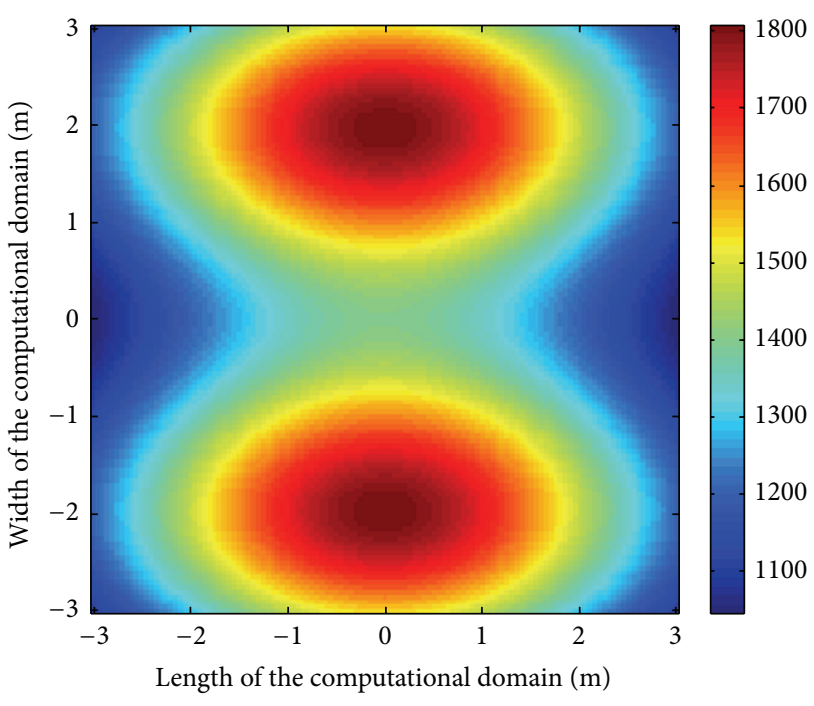

FIGURE 15: Temperature distribution reconstructed by the DRMC method when the noise variance and the sampling ratio are 9 and $33.06 \%$.

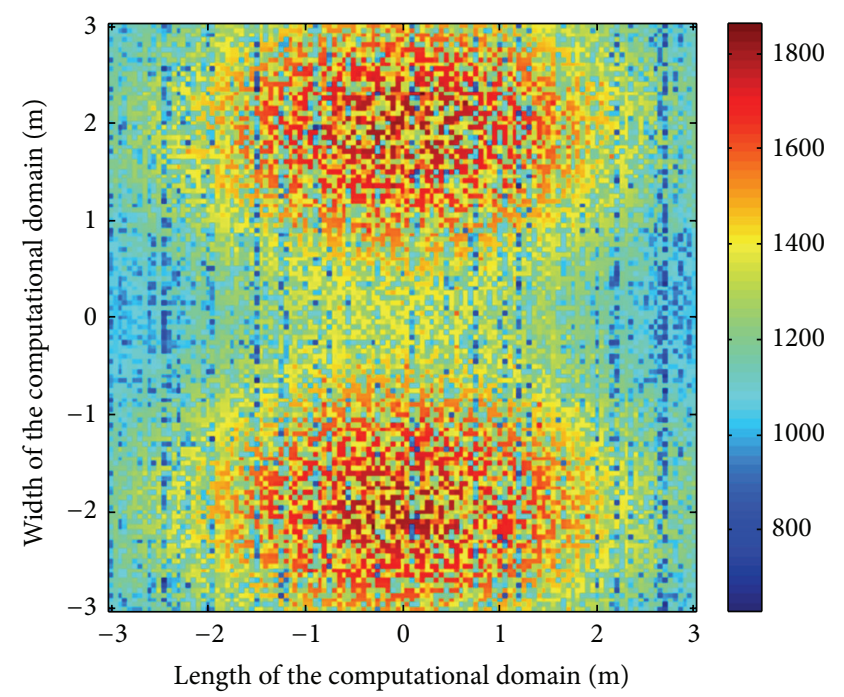

FIGURE 16: Temperature distribution reconstructed by the MC method when the noise variance and the sampling ratio are 9 and $33.06 \%$.

by the following equations from the partial temperature measurement data:

$$
\begin{gathered}
\frac{\partial T}{\partial t}=\frac{\lambda}{\rho c}\left(\frac{\partial^{2} T}{\partial x^{2}}+\frac{\partial^{2} T}{\partial y^{2}}\right) \\
T(x, y)=780, \quad \text { when } x=0,0 \leq y \leq 0.5 \\
T(x, y)=780, \quad \text { when } y=0.5,0 \leq x \leq 0.5 \\
T(x, y)=780, \quad \text { when } x=0.5,0 \leq y \leq 0.5 \\
T(x, y)=780, \quad \text { when } y=0,0 \leq x \leq 0.5 \\
T(x, y, 0)=320,
\end{gathered}
$$




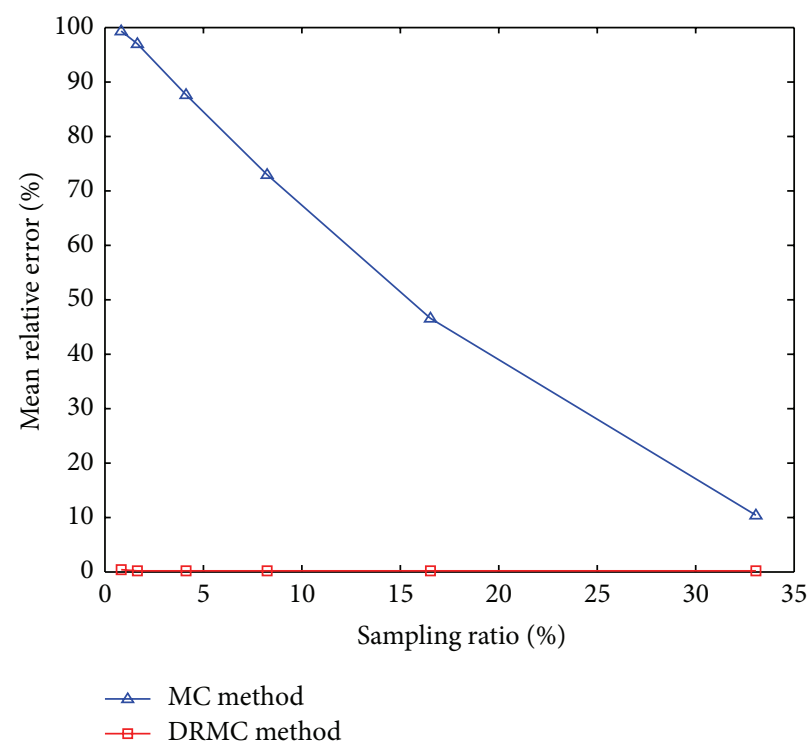

FIGURE 17: Mean relative errors under different sampling ratios.

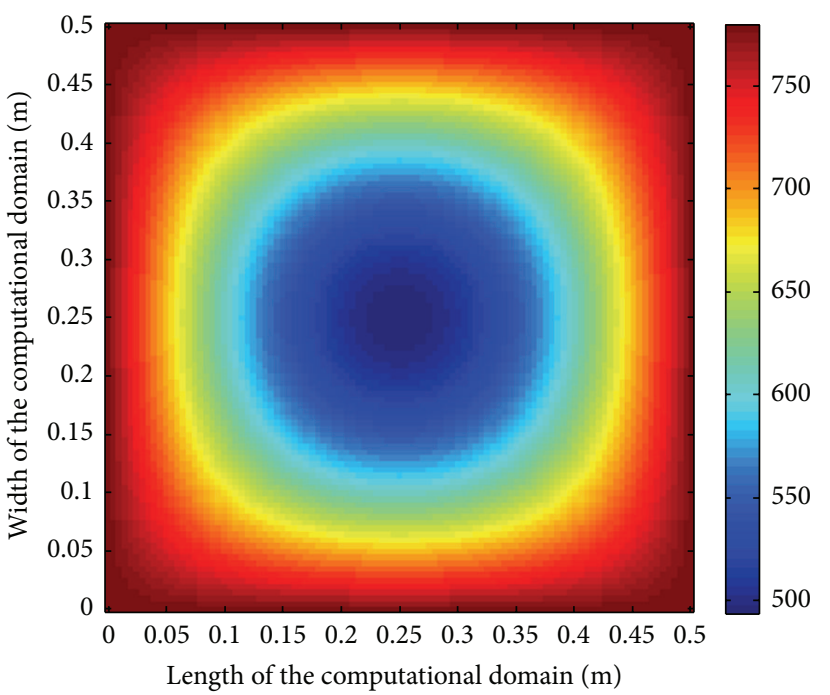

(a)

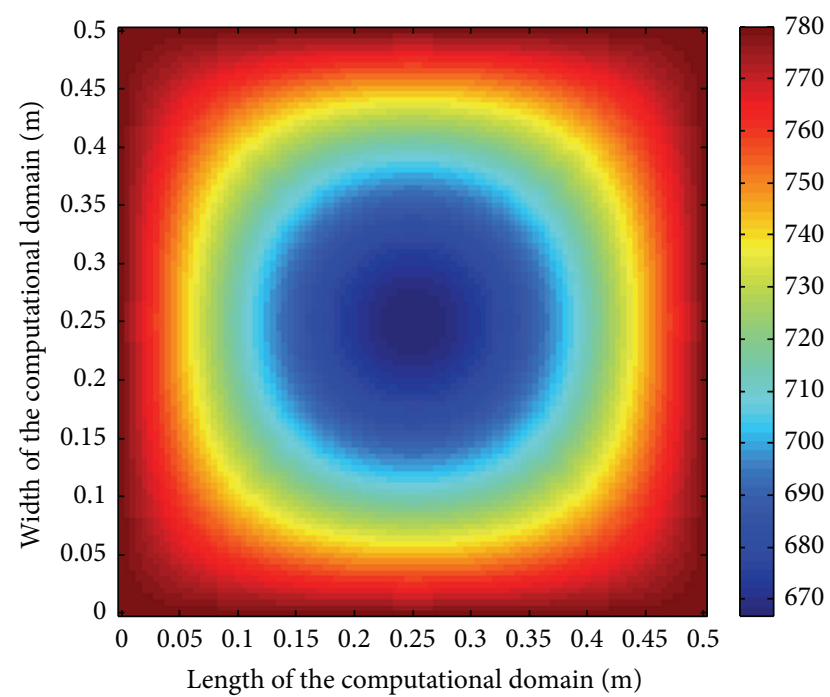

(b)

Figure 18: Original temperature distributions. (a) Original temperature distribution at $t=100 \mathrm{~s}$; (b) original temperature distribution at $t=200 \mathrm{~s}$.

where $\lambda=400 \mathrm{~W} /(\mathrm{m} \cdot \mathrm{K}), \rho=8700 \mathrm{~kg} / \mathrm{m}^{3}$, and $c=385 \mathrm{~J} /(\mathrm{kg}$. K).

Figure 18 shows the original temperature distributions solved by the FEM. In the MC method, $\alpha_{1}=50$. In the DRMC method, $\mathbf{W}_{k}=\operatorname{diag}\left(1 /\left|\mathbf{B}_{k-1, \tau, 1}\right|^{p}, 1 /\left|\mathbf{B}_{k-1, \tau, 2}\right|^{p}, \ldots\right.$, $\left.1 /\left|\mathbf{B}_{k-1, \tau, n}\right|^{p}\right), p=1.5, \mu_{1}=10^{-5}, \mu_{2}=10^{-5}, \mu_{3}=5$, $\mu_{4}=1, \mu_{5}=1 \times 10^{-7}$, and $\sigma^{2}=1$. The simulation data is used to serve as measurement data; the normal distribution random number with the variance of 9 is added to original data to simulate a real measurement environment. For fair comparison, in this case the known temperature data is randomly sampled. Figures 19 and 20 show the temperature distributions reconstructed by the DRMC method and the
MC algorithm when the sampling ratio and the noise variance are $19.80 \%$ and 9, respectively. Figure 21 presents the MREs of the DRMC method and the MC algorithm under different sampling ratios when the noise variance is 9 .

Figures 19 and 20 are the temperature distributions reconstructed by the DRMC method and the MC technique at different time instants when the sampling ratio and the noise variance are $19.80 \%$ and 9, respectively. As can be expected, for dynamic reconstruction cases, the results reconstructed by the DRMC method are better than the MC method. Additionally, it can be observed from Figures 21 and 22 that with the increasing of the sampling ratios the reconstruction quality of the both algorithms is gradually 


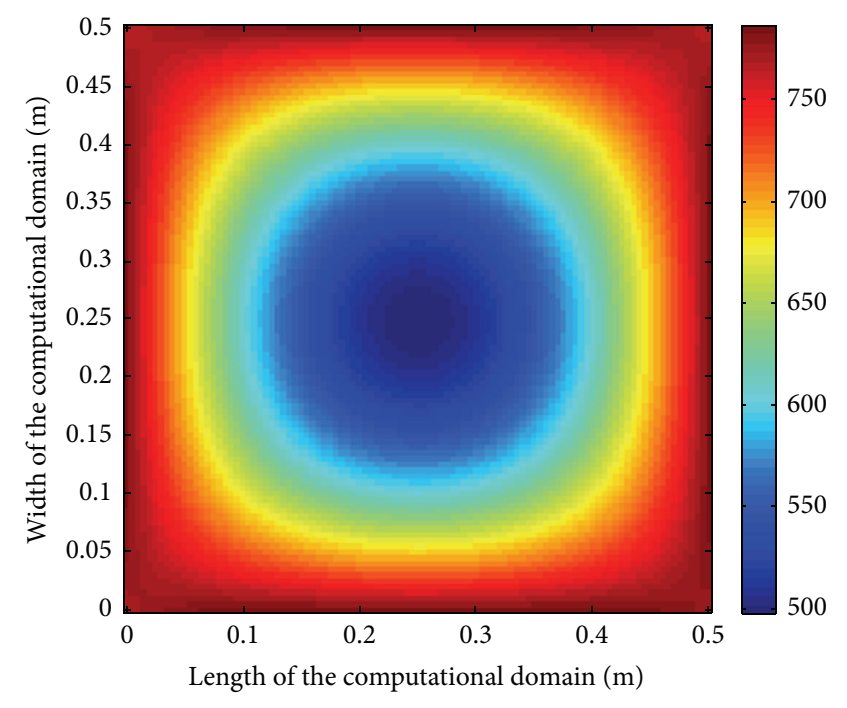

(a)

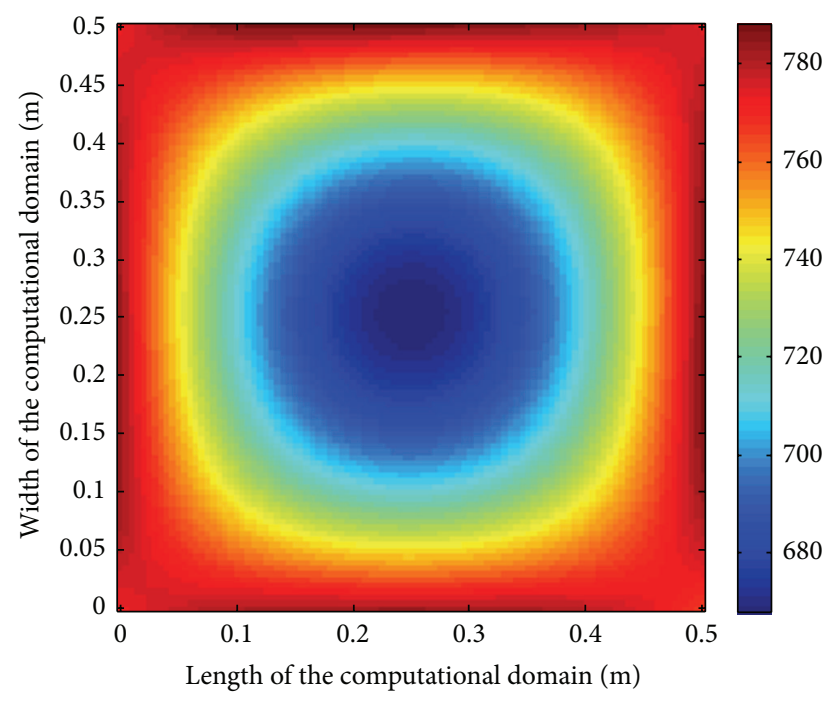

(b)

FIGURE 19: Temperature distributions reconstructed by the DRMC method when the noise variance and the sampling ratio are 9 and $19.80 \%$. (a) Reconstructed temperature distribution at $t=100 \mathrm{~s}$; (b) reconstructed temperature distribution at $t=200 \mathrm{~s}$.

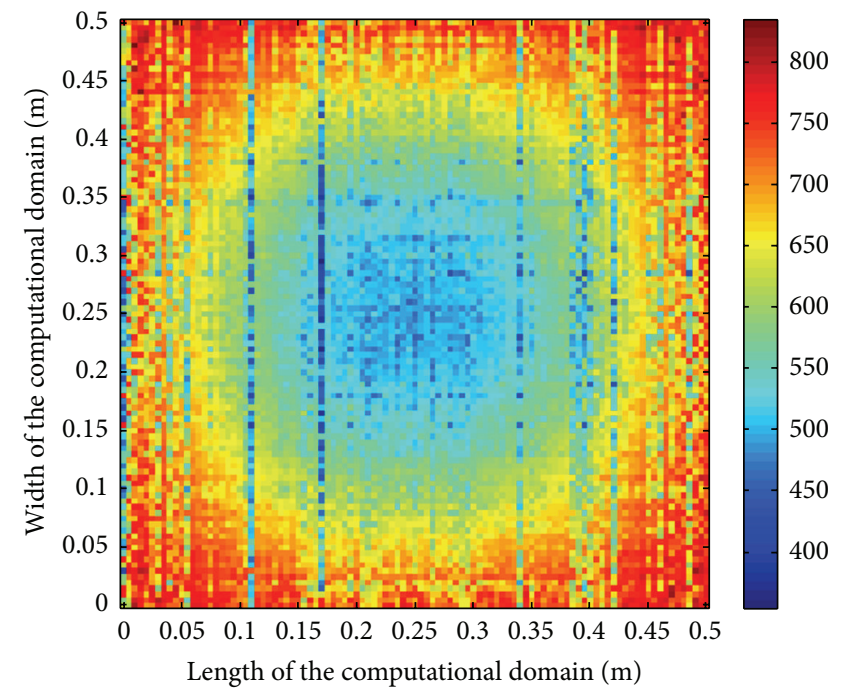

(a)

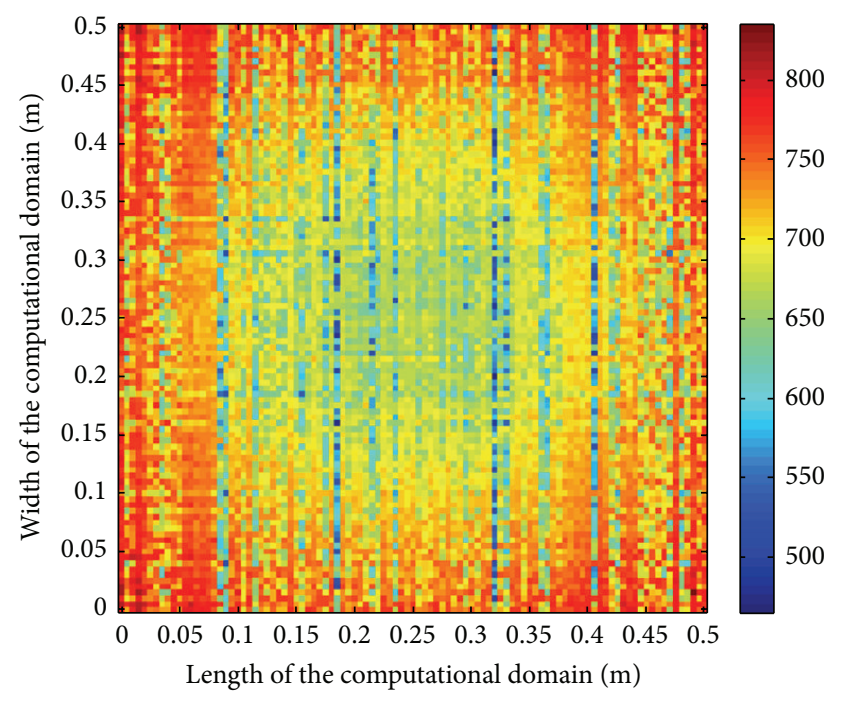

(b)

FIGURE 20: Temperature distributions reconstructed by the MC method when the noise variance and the sampling ratio are 9 and $19.80 \%$. (a) Reconstructed temperature distribution at $t=100 \mathrm{~s}$; (b) reconstructed temperature distribution at $t=200 \mathrm{~s}$.

improved. However, it can be seen that the reconstruction quality of the DRMC method is higher than that of the MC method when the sampling ratio is small.

Following the results and discussions presented in previous sections, it can be concluded that the DRMC algorithm is competent in implementing the FIS tasks. Additionally, in the illustrated cases, in order to evaluate the feasibility of the DRMC method, the measurement data are randomly sampled, and numerical results indicate that the final solutions are not sensitive to the locations of the measurement data. This feature is highly desired for real applications. In this paper, merely the temperature distribution cases are shown. It is worth emphasizing that the DRMC algorithm is a general framework for the FIS tasks, which may be useful for other related problems.

\section{Conclusions}

Acquiring the field information on temperature, pressure, concentration, or velocity is crucial for the monitoring of chemical reactors, multiphase flow systems, heat transfer units, atmospheric pollutant diffusion, and underground pollutant migration, and thus facilitates the improvement of the system efficiency, the energy saving, and the reduction of the pollutant emission. In this paper, a DRMC method, which 


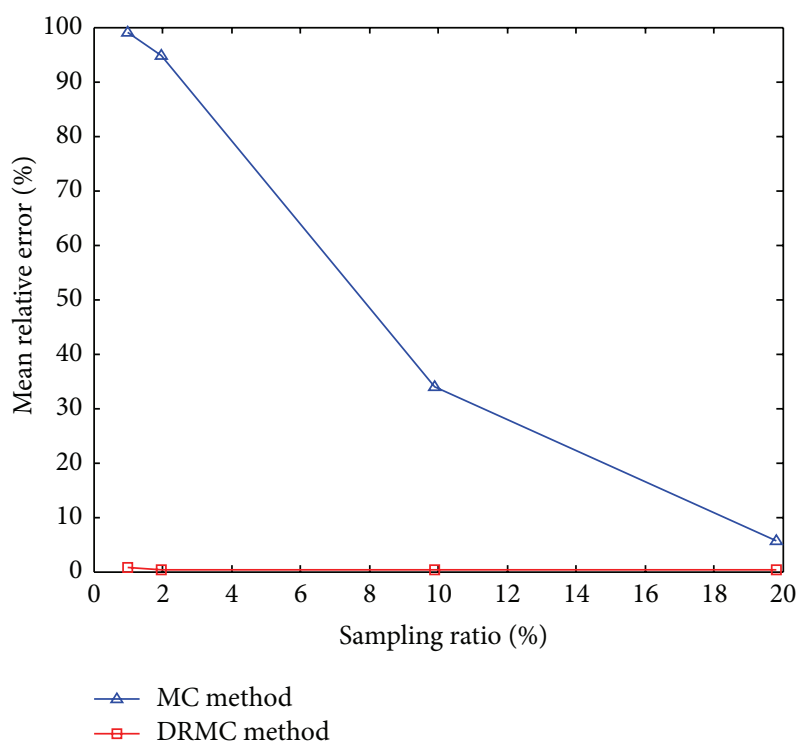

FIGURE 21: Mean relative errors under different sampling ratios at $t=100 \mathrm{~s}$.

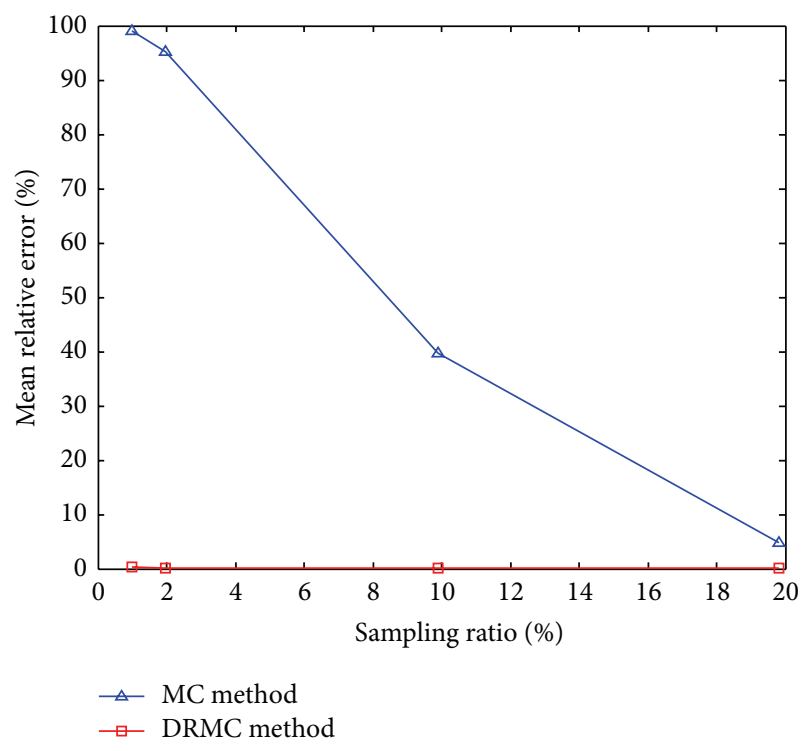

FIGURE 22: Mean relative errors under different sampling ratios at $t=200 \mathrm{~s}$.

incorporates the point measurement method and numerical optimization approach, is proposed for solving the FIS problem. Numerical simulations are implemented to validate the feasibility and effectiveness of the proposed algorithm. It is found that differing from common inverse problems, numerical simulation approaches, and tomography based field measurement methods, in the proposed method the field information is reconstructed without the knowledge on governing equations of the measurement objects, initial conditions, boundary conditions, and physical property parameters of materials, except the limited number of the measurement data. For the cases simulated in this paper, the reconstruction results from the proposed method are in a good agreement with the original distributions. Furthermore, the reconstruction results from the noise-contaminated data show that the proposed algorithm is robust to the inaccurate property on the measurement data. As a result, a promising method is introduced for the FIS problems.

It is worth mentioning that the proposed method provides a general framework for the FIS tasks, which may be useful for other related problems. However, studies indicate that each method may show different numerical performances to different FIS problems. In practice, the selection of an appropriate reconstruction method depends mainly on a specific reconstruction object and the modeling of the related prior information. This work provides an alternative approach for the FIS problem, which needs to be further validated by more cases in the future.

\section{Conflict of Interests}

The author declares that there is no conflict of interests regarding the publication of this paper.

\section{Acknowledgments}

The authors wish to thank the National Natural Science Foundation of China (no. 51206048), the Fundamental Research Funds for the Central Universities (no. 13MS11), and the Open Foundation from Key Laboratory of Efficient Utilization of Low and Medium Grade Energy (Tianjin University), Ministry of Education of China (no. 201301-301) for supporting this research.

\section{References}

[1] D. Graupe, Principles of Artificial Neural Networks, World Scientific Publishing, 2007.

[2] J. Lei and S. Liu, "Temperature field reconstruction from the partial measurement data using the gappy proper orthogonal decomposition," IET Science, Measurement and Technology, vol. 7, no. 3, pp. 171-179, 2013.

[3] D. Venturi and G. E. Karniadakis, "Gappy data and reconstruction procedures for flow past a cylinder," Journal of Fluid Mechanics, vol. 519, no. 2, pp. 315-336, 2004.

[4] K. Willcox, "Unsteady flow sensing and estimation via the gappy proper orthogonal decomposition," Computers \& Fluids, vol. 35, no. 2, pp. 208-226, 2006.

[5] A. Yakhot, T. Anor, and G. E. Karniadakis, "A reconstruction method for gappy and noisy arterial flow data," IEEE Transactions on Medical Imaging, vol. 26, no. 12, pp. 1681-1697, 2007.

[6] X. Yu, Y. Liu, Y. Zhu et al., "Efficient sampling and compressive sensing for urban monitoring vehicular sensor networks," IET Wireless Sensor Systems, vol. 2, no. 3, pp. 214-221, 2012.

[7] E. J. Candès and B. Recht, "Exact matrix completion via convex optimization," Foundations of Computational Mathematics, vol. 9, no. 6, pp. 717-772, 2009.

[8] E. J. Candès and T. Tao, “The power of convex relaxation: nearoptimal matrix completion," IEEE Transactions on Information Theory, vol. 56, no. 5, pp. 2053-2080, 2010. 
[9] S. Deng and Y. Hwang, "Solution of inverse heat conduction problems using Kalman filter-enhanced Bayesian back propagation neural network data fusion," International Journal of Heat and Mass Transfer, vol. 50, no. 11-12, pp. 2089-2100, 2007.

[10] A. P. Fernandes, P. F. B. Sousa, V. L. Borges, and G. Guimaraes, "Use of 3D-transient analytical solution based on Green's function to reduce computational time in inverse heat conduction problems," Applied Mathematical Modelling, vol. 34, no. 12, pp. 4040-4049, 2010.

[11] E. Hetmaniok, I. Nowak, D. Słota, and R. Wituła, "Application of the homotopy perturbation method for the solution of inverse heat conduction problem," International Communications in Heat and Mass Transfer, vol. 39, no. 1, pp. 30-35, 2012.

[12] C.-S. Liu, "A lie-group adaptive method to identify spatialdependence heat conductivity coefficients," Numerical Heat Transfer Part B: Fundamentals, vol. 60, no. 4, pp. 305-323, 2011.

[13] M. N. Ozisik and H. R. B. Orlande, Inverse Heat Transfer: Fundamentals and Applications, Taylor \& Francis, New York, NY, USA, 2000.

[14] N. Tian, J. Sun, W. Xu, and C.-H. Lai, "Estimation of unknown heat source function in inverse heat conduction problems using quantum-behaved particle swarm optimization," International Journal of Heat and Mass Transfer, vol. 54, no. 17-18, pp. 41104116, 2011.

[15] J. Wang and N. Zabaras, "A Bayesian inference approach to the inverse heat conduction problem," International Journal of Heat and Mass Transfer, vol. 47, no. 17-18, pp. 3927-3941, 2004.

[16] P. L. Woodfield and M. Monde, "Estimation of uncertainty in an analytical inverse heat conduction solution," Experimental Heat Transfer, vol. 22, no. 3, pp. 129-143, 2009.

[17] L. Kong, M. Xia, X. Liu et al., "Data loss and reconstruction in wireless sensor networks," IEEE Transactions on Parallel and Distributed Systems, vol. 25, no. 99, pp. 2818-2828, 2014.

[18] E. J. Candès and Y. Plan, "Matrix completion with noise," Proceedings of the IEEE, vol. 98, no. 6, pp. 925-936, 2010.

[19] C. Chen, B. He, and X. Yuan, "Matrix completion via an alternating direction method," IMA Journal of Numerical Analysis, vol. 32, no. 1, pp. 227-245, 2012.

[20] A. Majumdar and R. K. Ward, "Some empirical advances in matrix completion," Signal Processing, vol. 91, no. 5, pp. 13341338, 2011.

[21] A. Majumdar, "Improved dynamic MRI reconstruction by exploiting sparsity and rank-deficiency," Magnetic Resonance Imaging, vol. 31, no. 5, pp. 789-795, 2013.

[22] K.-C. Toh and S. Yun, "An accelerated proximal gradient algorithm for nuclear norm regularized least squares problems," Pacific Journal of Optimization, vol. 6, no. 3, pp. 615-640, 2010.

[23] Z. Wen, W. Yin, and Y. Zhang, "Solving a low-rank factorization model for matrix completion by a nonlinear successive overrelaxation algorithm," Mathematical Programming Computation, vol. 4, no. 4, pp. 333-361, 2012.

[24] G. Liu, Z. Lin, S. Yan, J. Sun, Y. Yu, and Y. Ma, "Robust recovery of subspace structures by low-rank representation," IEEE Transactions on Pattern Analysis and Machine Intelligence, vol. 35, no. 1, pp. 171-184, 2013.

[25] D. Yang, G. Liao, S. Zhu, X. Yang, and X. Zhang, "SAR imaging with undersampled data via matrix completion," IEEE Geoscience \& Remote Sensing Letters, vol. 11, no. 9, pp. 1539-1543, 2014.

[26] X. Liu, Meshfree Method, Science Press, Beijing, China, 2011.
[27] M. D. Butala, R. J. Hewett, R. A. Frazin, and F. Kamalabadi, "Dynamic three-dimensional tomography of the solar corona," Solar Physics, vol. 262, no. 2, pp. 495-509, 2010.

[28] A. N. Tikhonov and V. Y. Arsenin, Solution of Ill-Posed Problems, V.H. Winston \& Sons, New York, NY, USA, 1977.

[29] Y. F. Wang, Computational Methods for Inverse Problems and Their Applications, Higher Education Press, Beijing, China, 2007.

[30] T. Y. Xiao, S. G. Yu, and Y. F. Wang, Numerical Methods for Inverse Problems, Science Press, Beijing, China, 2003.

[31] M. Blondel, K. Seki, and K. Uehara, "Block coordinate descent algorithms for large-scale sparse multiclass classification," Machine Learning, vol. 93, no. 1, pp. 31-52, 2013.

[32] T. F. Chan and C. K. Wong, "Convergence of the alternating minimization algorithm for blind deconvolution," Linear Algebra and Its Applications, vol. 316, no. 1-3, pp. 259-285, 2000.

[33] E. Kim, M. Lee, C. H. Choi, N. Kwak, and S. Oh, "Efficient $1_{1}$-norm-based low-rank matrix approximations for large-scale problems using alternating rectified gradient method," IEEE Transactions on Neural Networks and Learning Systems, 2014.

[34] X.-G. Lv, Y.-Z. Song, S.-X. Wang, and J. Le, "Image restoration with a high-order total variation minimization method," Applied Mathematical Modelling: Simulation and Computation for Engineering and Environmental Systems, vol. 37, no. 16-17, pp. 8210-8224, 2013.

[35] Z. Qin, K. Scheinberg, and D. Goldfarb, "Efficient blockcoordinate descent algorithms for the group Lasso," Mathematical Programming Computation, vol. 5, no. 2, pp. 143-169, 2013.

[36] P. Tseng, "Convergence of a block coordinate descent method for nondifferentiable minimization," Journal of Optimization Theory and Applications, vol. 109, no. 3, pp. 475-494, 2001.

[37] Y. Xiao, T. Zeng, J. Yu, and M. K. Ng, "Restoration of images corrupted by mixed Gaussian-impulse noise via $L_{1}-L_{0}$ minimization," Pattern Recognition, vol. 44, no. 8, pp. 1708-1720, 2011.

[38] J.-F. Cai, S. Osher, and Z. Shen, "Split bregman methods and frame based image restoration," Multiscale Modeling \& Simulation, vol. 8, no. 2, pp. 337-369, 2009.

[39] T. Goldstein and S. Osher, "The split Bregman method for L1regularized problems," SIAM Journal on Imaging Sciences, vol. 2, no. 2, pp. 323-343, 2009.

[40] T. Goldstein, X. Bresson, and S. Osher, "Geometric applications of the split Bregman method: segmentation and surface reconstruction," Journal of Scientific Computing, vol. 45, no. 1-3, pp. 272-293, 2010.

[41] H. Gao, J.-F. Cai, Z. Shen, and H. Zhao, "Robust principal component analysis-based four-dimensional computed tomography," Physics in Medicine and Biology, vol. 56, no. 11, pp. 31813198, 2011.

[42] W. Yin, S. Osher, D. Goldfarb, and J. Darbon, "Bregman iterative algorithms for $l_{1}$-minimization with applications to compressed sensing," SIAM Journal on Imaging Sciences, vol. 1, no. 1, pp. 143$168,2008$.

[43] X. Zhang, M. Burger, X. Bresson, and S. Osher, "Bregmanized nonlocal regularization for deconvolution and sparse reconstruction," SIAM Journal on Imaging Sciences, vol. 3, no. 3, pp. 253-276, 2010.

[44] X. Zhang, M. Burger, and S. Osher, "A unified primal-dual algorithm framework based on Bregman iteration," Journal of Scientific Computing, vol. 46, no. 1, pp. 20-46, 2011. 
[45] J.-F. Cai, E. J. Candes, and Z. Shen, "A singular value thresholding algorithm for matrix completion," SIAM Journal on Optimization, vol. 20, no. 4, pp. 1956-1982, 2010.

[46] Z. L. Li, Temperature field reconstruction based on acoustic tomography [M.S. thesis], Shenyang University of Technology, Shenyang, China, 2007. 


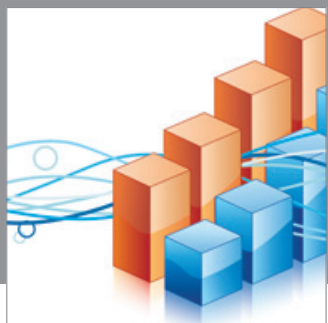

Advances in

Operations Research

mansans

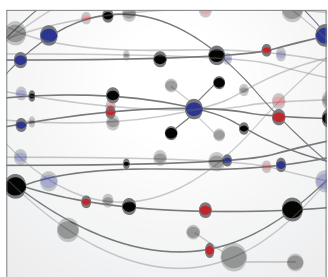

The Scientific World Journal
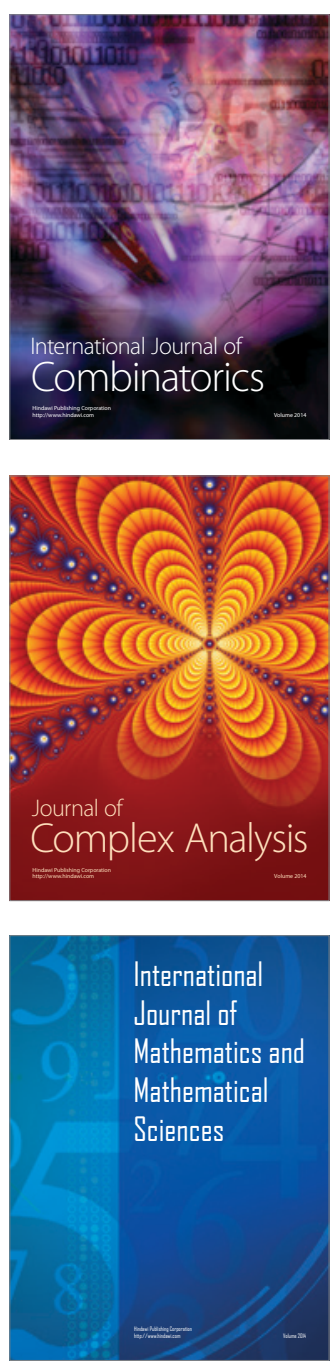
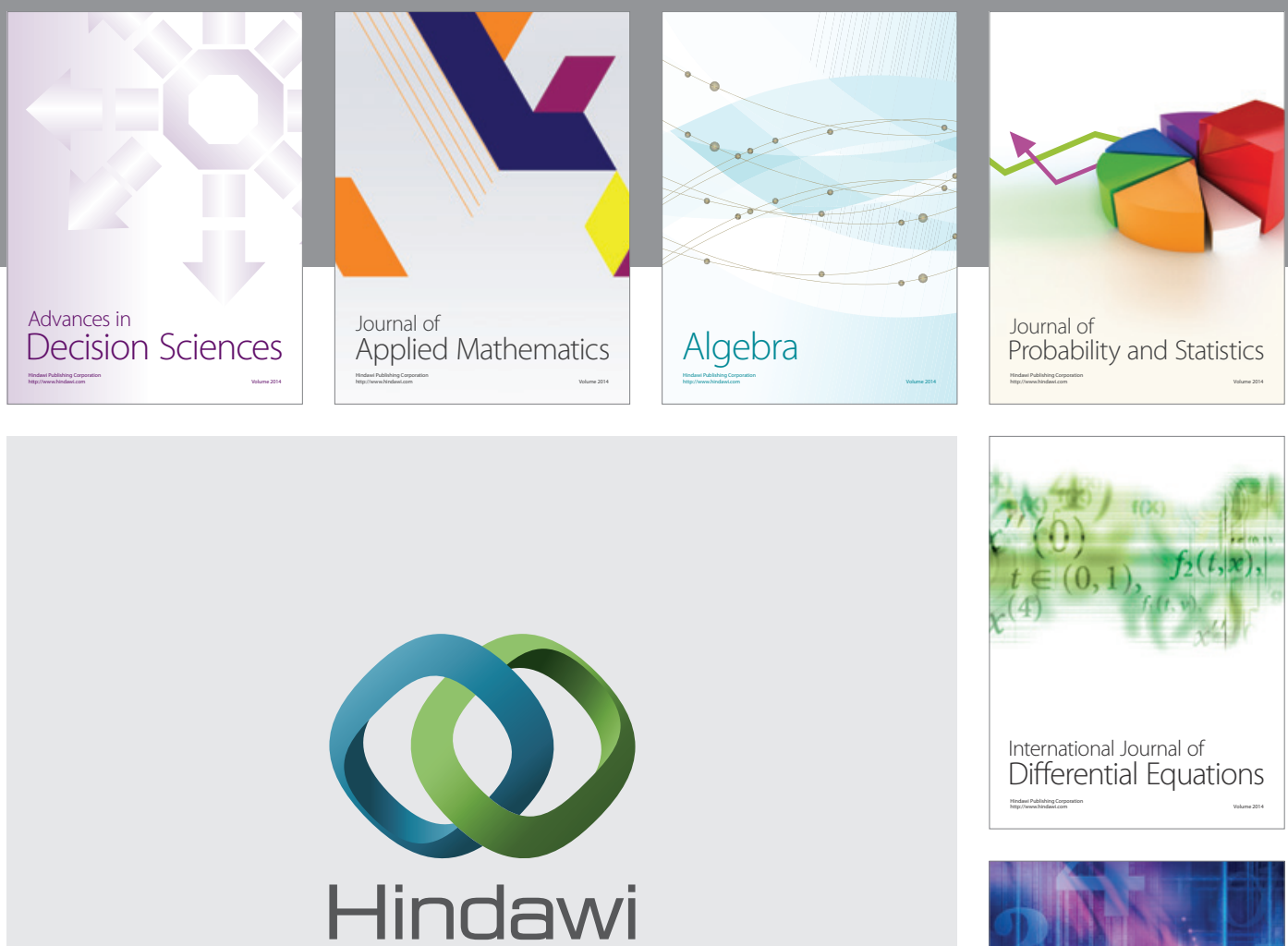

Submit your manuscripts at http://www.hindawi.com
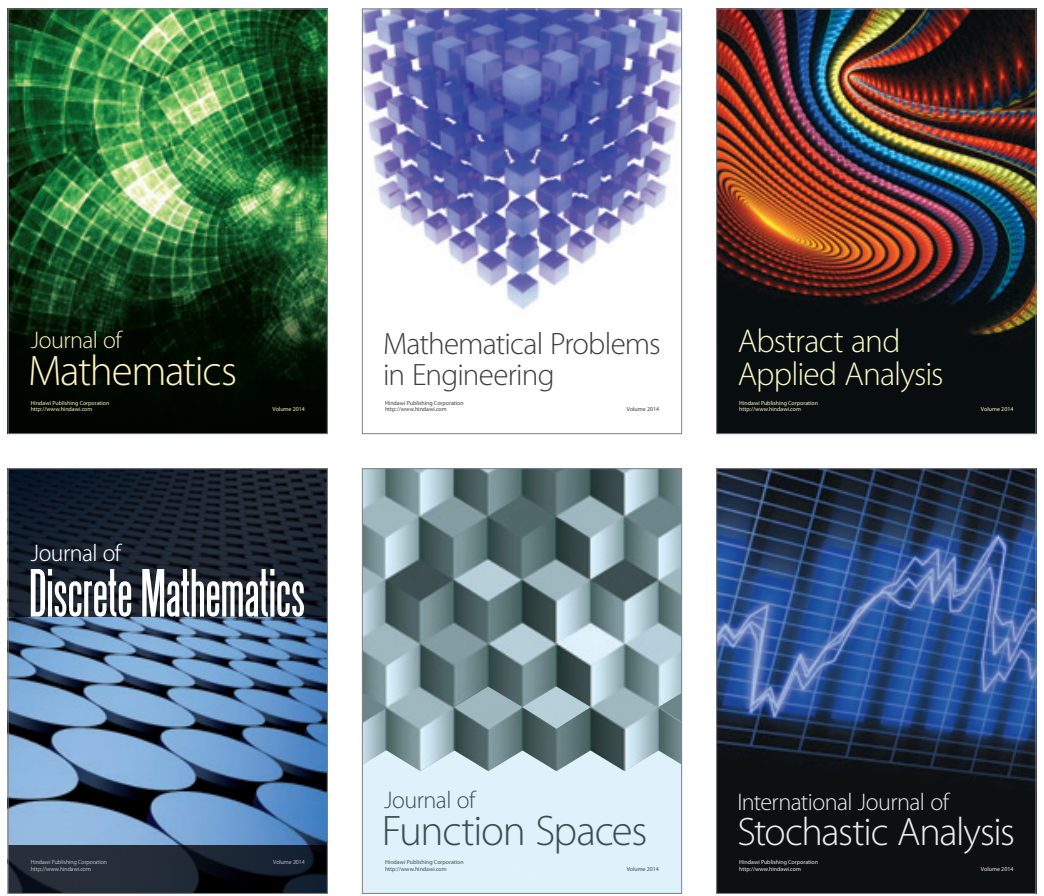

Journal of

Function Spaces

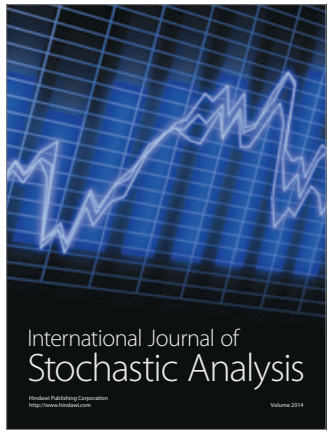

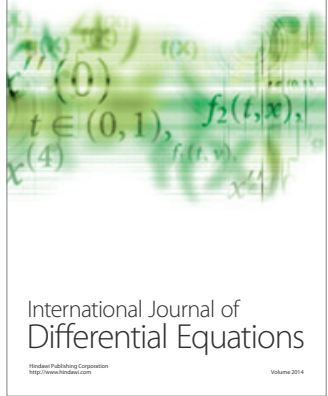
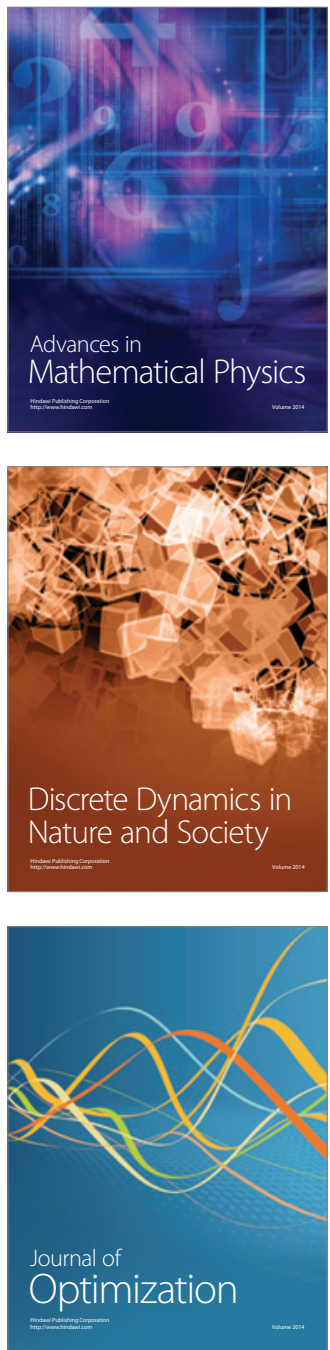